\title{
APPENDIX 13
}

\section{Equations for Tree Height, Crown Height, and Crown Width}

Equations to estimate tree height (Table 30), crown height (Table 31), and crown width (Table 32) are based on diameter at breast height (d.b.h.).

Crown height is the height of the crown from the lowest part of the crown to the top of the crown. These equations are used when only d.b.h. is measured and in population projections to estimate leaf area. These formulas were developed by Robert Bridges and John Stanovick in collaboration with the i-Tree Team. DBHMin and DBHMax are the minimum and maximum d.b.h. values, respectively, to be used with the equations. D.b.h. is in inches; height and width outputs are in feet.

Table 30.-Tree height equations

\begin{tabular}{|c|c|c|c|c|c|c|c|}
\hline $\begin{array}{l}\text { Taxonomic } \\
\text { Class }\end{array}$ & Taxon & DBHMin & DBHMax & Model & BO & B1 & B2 \\
\hline Order & Cornales & 1.00 & 27.20 & $B O+\left(D B H^{*} B 1\right)+\left(D B H^{2} * B 2\right)$ & 8.623 & 4.2058 & -0.0629 \\
\hline Order & Dipsacales & 1.00 & 12.40 & $e^{\left(B O+\left(\ln (D B H)^{*} B 1\right)\right)}$ & 2.4026 & 0.1627 & \\
\hline Order & Ebenales & 1.00 & 16.10 & $e^{(B O+(\ln (D B H) * B 1))}$ & 1.9989 & 0.7689 & \\
\hline Order & Ericales & 1.00 & 7.00 & $B 0+(D B H * B 1)$ & 11.9413 & 4.2381 & \\
\hline Order & Euphorbiales & 1.00 & 29.30 & $e^{(B O+(\ln (D B H) * B 1))}$ & 2.628 & 0.3701 & \\
\hline Order & Fabales & 1.00 & 46.20 & $e^{(B O+(\ln (D B H) * B 1))}$ & 2.46 & 0.5804 & \\
\hline Order & Fagales & 1.00 & 31.00 & $B 0+\left(D B H^{*} B 1\right)+\left(D B H^{2} * B 2\right)$ & 11.2564 & 4.61 & -0.0735 \\
\hline Order & Gentianales & 1.00 & 16.50 & $B 0+\left(D B H^{*} B 1\right)$ & 10.1716 & 1.9588 & \\
\hline Order & Hamamelidales & 1.00 & 25.00 & $B O+\left(D B H^{*} B 1\right)+\left(D B H^{2} * B 2\right)$ & 11.3341 & 5.5504 & -0.1127 \\
\hline Order & Juglandales & 1.00 & 24.00 & $B 0+\left(D B H^{*} B 1\right)+\left(D B H^{2} * B 2\right)$ & 9.5467 & 5.7118 & -0.1189 \\
\hline Order & Laurales & 1.00 & 17.00 & $B 0+\left(D B H^{*} B 1\right)+\left(D B H^{2} * B 2\right)$ & 5.5344 & 6.3008 & -0.1808 \\
\hline Order & Magnoliales & 1.00 & 34.00 & $B 0+\left(D B H^{*} B 1\right)+\left(D B H^{2} * B 2\right)$ & 12.4282 & 5.3742 & -0.0792 \\
\hline Order & Malvales & 1.00 & 40.00 & $e^{(B O+(\ln (D B H) * B 1))}$ & 2.5694 & 0.4278 & \\
\hline Order & Myrtales & 1.00 & 101.60 & $e^{\left(B O+\left(\ln (D B H)^{*} B 1\right)\right)}$ & 2.6401 & 0.4236 & \\
\hline Order & Pinales & 1.30 & 28.00 & $B 0+\left(D B H^{*} B 1\right)+\left(D B H^{2} * B 2\right)$ & 0.7293 & 5.4819 & -0.0988 \\
\hline Order & Rhamnales & 1.00 & 16.40 & $e^{(B O+(\ln (D B H) * B 1))}$ & 2.603 & 0.204 & \\
\hline Order & Rosales & 1.00 & 28.00 & $B 0+\left(D B H^{*} B 1\right)+\left(D B H^{2} * B 2\right)$ & 8.1664 & 3.729 & -0.066 \\
\hline Order & Salicales & 1.00 & 23.00 & $B 0+\left(D B H^{*} B 1\right)+\left(D B H^{2} * B 2\right)$ & 5.4057 & 5.6113 & -0.1238 \\
\hline Order & Sapindales & 1.00 & 70.90 & $e^{\left(B O+\left(\ln (D B H)^{*} B 1\right)\right)}$ & 2.564 & 0.5532 & \\
\hline Order & Scrophulariales & 1.00 & 44.00 & $e^{(B 0+(\ln (D B H) * B 1))}$ & 2.6073 & 0.5432 & \\
\hline Order & Urticales & 1.00 & 58.00 & $e^{(B 0+(\ln (D B H) * B 1))}$ & 2.4549 & 0.547 & \\
\hline Family & Aceraceae & 1.00 & 70.90 & $e^{(B O+(\ln (D B H) * B 1))}$ & 2.6269 & 0.5275 & \\
\hline Family & Anacardiaceae & 1.00 & 9.80 & $e^{(B O+(\ln (D B H) * B 1))}$ & 2.3661 & 0.4619 & \\
\hline Family & Arecaceae & 3.50 & 38.00 & $B 0+(D B H * B 1)$ & 5.0976 & 0.8852 & \\
\hline Family & Betulaceae & 1.00 & 30.00 & $e^{(B 0+(\ln (D B H) * B 1))}$ & 2.5904 & 0.5756 & \\
\hline Family & Bignoniaceae & 1.00 & 35.50 & $e^{(B O+(\ln (D B H) * B 1))}$ & 2.444 & 0.5281 & \\
\hline
\end{tabular}


Table 30.-Continued

\begin{tabular}{|c|c|c|c|c|c|c|c|}
\hline $\begin{array}{l}\text { Taxonomic } \\
\text { Class }\end{array}$ & Taxon & DBHMin & DBHMax & Model & BO & B1 & B2 \\
\hline Family & Cornaceae & 1.00 & 21.30 & $e^{(B O+(\ln (D B H) * B 1))}$ & 2.5895 & 0.317 & \\
\hline Family & Cupressaceae & 1.00 & 61.00 & $e^{(B O+(\ln (D B H) * B 1))}$ & 1.9729 & 0.5997 & \\
\hline Family & Euphorbiaceae & 1.00 & 29.30 & $e^{\left(B O+\left(\ln (D B H)^{*} B 1\right)\right)}$ & 2.6269 & 0.3717 & \\
\hline Family & Fagaceae & 1.00 & 32.00 & $B 0+\left(D B H^{*} B 1\right)+\left(D B H^{2} * B 2\right)$ & 11.3008 & 4.6004 & -0.0726 \\
\hline Family & Hamamelidaceae & 1.00 & 23.00 & $B 0+\left(D B H^{*} B 1\right)+\left(D B H^{2} * B 2\right)$ & 9.8581 & 6.1332 & -0.1338 \\
\hline Family & Lauraceae & 1.00 & 18.00 & $B 0+\left(D B H^{*} B 1\right)+\left(D B H^{2} * B 2\right)$ & 5.5344 & 6.3008 & -0.1808 \\
\hline Family & Lythraceae & 1.00 & 13.00 & $e^{\left(B O+\left(\ln (D B H)^{*} B 1\right)\right)}$ & 2.576 & 0.2281 & \\
\hline Family & Magnoliaceae & 1.00 & 34.00 & $B 0+\left(D B H^{*} B 1\right)+\left(D B H^{2} * B 2\right)$ & 12.4815 & 5.3673 & -0.079 \\
\hline Family & Moraceae & 1.00 & 58.00 & $e^{(B O+(\ln (D B H) * B 1))}$ & 2.4045 & 0.4945 & \\
\hline Family & Myrtaceae & 2.00 & 101.60 & $e^{(B 0+(\ln (D B H) * B 1))}$ & 2.6828 & 0.457 & \\
\hline Family & Nyssaceae & 1.00 & 23.00 & $B 0+\left(D B H^{*} B 1\right)+\left(D B H^{2} * B 2\right)$ & 5.8481 & 6.5082 & -0.1445 \\
\hline Family & Oleaceae & 1.00 & 44.00 & $e^{(B O+(\ln (D B H) * B 1))}$ & 2.6141 & 0.5505 & \\
\hline Family & Pinaceae & 1.30 & 27.00 & $B 0+\left(D B H^{*} B 1\right)+\left(D B H^{2} * B 2\right)$ & 1.2686 & 5.7342 & -0.1073 \\
\hline Family & Platanaceae & 1.00 & 51.20 & $e^{\left(B O+\left(\ln (D B H)^{*} B 1\right)\right)}$ & 2.5495 & 0.5319 & \\
\hline Family & Rhamnaceae & 1.00 & 16.40 & $e^{(B 0+(\ln (D B H) * B 1))}$ & 2.6043 & 0.214 & \\
\hline Family & Rosaceae & 1.00 & 28.00 & $B 0+\left(D B H^{*} B 1\right)+\left(D B H^{2} * B 2\right)$ & 8.1208 & 3.766 & -0.0678 \\
\hline Family & Rutaceae & 1.00 & 17.00 & $e^{(B O+(\ln (D B H) * B 1))}$ & 2.6784 & 0.1675 & \\
\hline Family & Scrophulariaceae & 1.00 & 23.00 & $B 0+(\ln (D B H) * B 1)$ & 6.8028 & 13.064 & \\
\hline Family & Simaroubaceae & 1.00 & 18.00 & $B 0+\left(D B H^{*} B 1\right)+\left(D B H^{2} * B 2\right)$ & 7.3852 & 5.0019 & -0.1373 \\
\hline Family & Tiliaceae & 1.00 & 40.00 & $e^{(B O+(\ln (D B H) * B 1))}$ & 2.5996 & 0.4173 & \\
\hline Family & Ulmaceae & 1.00 & 38.00 & $e^{(B 0+(\ln (D B H) * B 1))}$ & 2.4743 & 0.56 & \\
\hline Genus & Betula & 1.00 & 30.00 & $e^{(B 0+(\ln (D B H) * B 1))}$ & 2.6637 & 0.5156 & \\
\hline Genus & Carpinus & 1.00 & 15.20 & $e^{\left(B O+\left(\ln (D B H)^{*} B 1\right)\right)}$ & 2.7671 & 0.3205 & \\
\hline Genus & Carya & 1.00 & 24.00 & $B 0+\left(D B H^{*} B 1\right)+\left(D B H^{2 *} B 2\right)$ & 9.1062 & 6.0882 & -0.1281 \\
\hline Genus & Catalpa & 1.00 & 35.50 & $e^{(B O+(\ln (D B H) * B 1))}$ & 2.4327 & 0.5463 & \\
\hline Genus & Celtis & 1.00 & 25.10 & $e^{\left(B O+\left(\ln (D B H)^{*} B 1\right)\right)}$ & 2.5054 & 0.5355 & \\
\hline Genus & Crataegus & 1.00 & 19.70 & $B 0+(\ln (D B H) * B 1)$ & 9.9227 & 6.9489 & \\
\hline Genus & Cupressus & 2.40 & 61.00 & $e^{(B O+(\ln (D B H) * B 1))}$ & 2.2087 & 0.5194 & \\
\hline Genus & Eucalyptus & 2.00 & 101.60 & $e^{(B O+(\ln (D B H) * B 1))}$ & 2.6662 & 0.4861 & \\
\hline Genus & Fagus & 1.00 & 27.00 & $B 0+\left(D B H^{*} B 1\right)+\left(D B H^{2} * B 2\right)$ & 8.7068 & 5.9258 & -0.1087 \\
\hline Genus & Fraxinus & 1.00 & 44.00 & $e^{(B O+(\ln (D B H) * B 1))}$ & 2.6408 & 0.5606 & \\
\hline Genus & Gleditsia & 1.30 & 46.20 & $e^{(B 0+(\ln (D B H) * B 1))}$ & 2.2877 & 0.5429 & \\
\hline Genus & Juglans & 1.00 & 33.00 & $e^{(B 0+(\ln (D B H) * B 1))}$ & 2.437 & 0.6096 & \\
\hline Genus & Juniperus & 1.00 & 23.40 & $e^{(B O+(\ln (D B H) * B 1))}$ & 2.1482 & 0.6457 & \\
\hline Genus & Liquidambar & 1.00 & 23.00 & $B 0+\left(D B H^{*} B 1\right)+\left(D B H^{2} * B 2\right)$ & 10.0766 & 6.0952 & -0.1325 \\
\hline Genus & Liriodendron & 1.00 & 52.00 & $B 0+(\ln (D B H) * B 1)$ & 4.1588 & 27.6253 & \\
\hline Genus & Magnolia & 1.00 & 50.30 & $e^{(B O+(\ln (D B H) * B 1))}$ & 2.6281 & 0.3999 & \\
\hline Genus & Malus & 1.00 & 29.00 & $e^{\left(B 0+\left(\ln (D B H)^{*} B 1\right)\right)}$ & 2.4015 & 0.2916 & \\
\hline Genus & Morus & 1.00 & 43.10 & $e^{\left(B O+\left(\ln (D B H)^{*} B 1\right)\right)}$ & 2.4279 & 0.4837 & \\
\hline Genus & Ostrya & 1.00 & 13.80 & $e^{\left(B 0+\left(\ln (D B H)^{*} B 1\right)\right)}$ & 2.5763 & 0.6192 & \\
\hline Genus & Phellodendron & 1.00 & 17.00 & $B 0+\left(D B H^{*} B 1\right)$ & 12.0006 & 2.4973 & \\
\hline Genus & Picea & 1.00 & 27.10 & $e^{(B 0+(\ln (D B H) * B 1))}$ & 1.9897 & 0.7152 & \\
\hline
\end{tabular}


Table 30.-Continued

\begin{tabular}{|c|c|c|c|c|c|c|c|}
\hline $\begin{array}{l}\text { Taxonomic } \\
\text { Class }\end{array}$ & Taxon & DBHMin & DBHMax & Model & BO & B1 & B2 \\
\hline Genus & Pinus & 1.00 & 28.00 & $B 0+\left(D B H^{*} B 1\right)+\left(D B H^{2} * B 2\right)$ & 2.4132 & 5.9575 & -0.1103 \\
\hline Genus & Populus & 1.00 & 24.00 & $B 0+\left(D B H^{*} B 1\right)+\left(D B H^{2} * B 2\right)$ & 4.8743 & 5.8071 & -0.119 \\
\hline Genus & Prunus & 1.00 & 24.00 & $B 0+\left(D B H^{*} B 1\right)+\left(D B H^{2} * B 2\right)$ & 6.9939 & 4.8184 & -0.1006 \\
\hline Genus & Pyrus & 1.00 & 19.40 & $B 0+\left(D B H^{*} B 1\right)$ & 10.7232 & 1.5314 & \\
\hline Genus & Quercus & 1.00 & 33.00 & $B 0+\left(D B H^{*} B 1\right)+\left(D B H^{2} * B 2\right)$ & 11.675 & 4.4321 & -0.0678 \\
\hline Genus & Rhus & 1.00 & 9.80 & $e^{\left(B O+\left(\ln (D B H)^{*} B 1\right)\right)}$ & 2.3635 & 0.4713 & \\
\hline Genus & Robinia & 1.00 & 35.00 & $e^{(B O+(\ln (D B H) * B 1))}$ & 2.477 & 0.624 & \\
\hline Genus & Salix & 1.00 & 41.30 & $e^{(B O+(\ln (D B H) * B 1))}$ & 2.4928 & 0.5078 & \\
\hline Genus & Sassafras & 1.00 & 19.00 & $B 0+\left(D B H^{*} B 1\right)+\left(D B H^{2} * B 2\right)$ & 5.9816 & 6.2457 & -0.1651 \\
\hline Genus & Syringa & 1.00 & 10.10 & $e^{\left(B O+\left(\ln (D B H)^{*} B 1\right)\right)}$ & 2.5968 & 0.1533 & \\
\hline Genus & Thuja & 1.00 & 35.60 & $B 0+(D B H * B 1)$ & 5.5987 & 2.2494 & \\
\hline Genus & Tsuga & 1.10 & 25.00 & $B 0+\left(D B H^{*} B 1\right)+\left(D B H^{2} * B 2\right)$ & 1.1086 & 5.1392 & -0.1033 \\
\hline Genus & Ulmus & 1.00 & 38.00 & $e^{\left(B O+\left(\ln (D B H)^{*} B 1\right)\right)}$ & 2.4772 & 0.5612 & \\
\hline Genus & Viburnum & 1.00 & 9.50 & $e^{(B O+(\ln (D B H) * B 1))}$ & 2.4026 & 0.1505 & \\
\hline Species & Acer negundo & 1.00 & 38.40 & $e^{(B O+(\ln (D B H) * B 1))}$ & 2.479 & 0.5371 & \\
\hline Species & Acer palmatum & 1.00 & 23.20 & $e^{(B 0+(\ln (D B H) * B 1))}$ & 2.0076 & 0.4723 & \\
\hline Species & Acer platanoides & 1.00 & 37.60 & $e^{(B 0+(\ln (D B H) * B 1))}$ & 2.6043 & 0.4534 & \\
\hline Species & Acer pseudoplatanus & 1.00 & 53.20 & $e^{(B O+(\ln (D B H) * B 1))}$ & 2.5842 & 0.54 & \\
\hline Species & Acer rubrum & 1.00 & 45.00 & $e^{(B O+(\ln (D B H) * B 1))}$ & 2.6393 & 0.5613 & \\
\hline Species & Acer saccharinum & 1.00 & 70.90 & $e^{\left(B 0+\left(\ln (D B H)^{*} B 1\right)\right)}$ & 2.69 & 0.4652 & \\
\hline Species & Acer saccharum & 1.00 & 40.00 & $B 0+(\ln (D B H) * B 1)$ & 11.3449 & 19.5311 & \\
\hline Species & Betula papyrifera & 1.00 & 21.30 & $e^{(B O+(\ln (D B H) * B 1))}$ & 2.8698 & 0.4381 & \\
\hline Species & Betula pendula & 1.00 & 30.00 & $B 0+(\ln (D B H) * B 1)$ & 17.7323 & 10.4284 & \\
\hline Species & Betula populifolia & 1.00 & 12.20 & $e^{\left(B O+\left(\ln (D B H)^{*} B 1\right)\right)}$ & 2.575 & 0.5303 & \\
\hline Species & Carpinus caroliniana & 1.00 & 10.40 & $e^{(B O+(\ln (D B H) * B 1))}$ & 2.783 & 0.3189 & \\
\hline Species & Carya alba & 1.00 & 22.00 & $B 0+\left(D B H^{*} B 1\right)+\left(D B H^{2} * B 2\right)$ & 9.3934 & 6.5955 & -0.1521 \\
\hline Species & Carya cordiformis & 1.00 & 33.00 & $B 0+\left(D B H^{*} B 1\right)+\left(D B H^{2} * B 2\right)$ & 6.4104 & 6.3745 & -0.083 \\
\hline Species & Carya glabra & 1.00 & 23.90 & $e^{\left(B O+\left(\ln (D B H)^{*} B 1\right)\right)}$ & 2.6764 & 0.5947 & \\
\hline Species & Carya illinoinensis & 1.50 & 35.00 & $e^{(B 0+(\ln (D B H) * B 1))}$ & 2.7997 & 0.4137 & \\
\hline Species & Carya ovata & 1.00 & 25.20 & $e^{(B O+(\ln (D B H) * B 1))}$ & 2.6684 & 0.6181 & \\
\hline Species & Catalpa speciosa & 1.00 & 35.50 & $e^{(B O+(\ln (D B H) * B 1))}$ & 2.4414 & 0.5456 & \\
\hline Species & Celtis laevigata & 1.30 & 25.00 & $e^{\left(B O+\left(\ln (D B H)^{*} B 1\right)\right)}$ & 2.6831 & 0.4544 & \\
\hline Species & Celtis occidentalis & 1.00 & 25.10 & $e^{(B 0+(\ln (D B H) * B 1))}$ & 2.3792 & 0.6343 & \\
\hline Species & Cornus florida & 1.00 & 17.60 & $e^{(B O+(\ln (D B H) * B 1))}$ & 2.6208 & 0.3129 & \\
\hline Species & Eucalyptus globulus & 2.00 & 101.60 & $e^{\left(B O+\left(\ln (D B H)^{*} B 1\right)\right)}$ & 2.6534 & 0.5052 & \\
\hline Species & Fagus grandifolia & 1.00 & 27.00 & $B 0+\left(D B H^{*} B 1\right)+\left(D B H^{2} * B 2\right)$ & 8.68 & 5.9536 & -0.1094 \\
\hline Species & Fraxinus americana & 1.00 & 22.00 & $B 0+\left(D B H^{*} B 1\right)+\left(D B H^{2} * B 2\right)$ & 9.0318 & 6.2878 & -0.1428 \\
\hline Species & $\begin{array}{l}\text { Fraxinus } \\
\text { pennsylvanica }\end{array}$ & 1.00 & 40.00 & $e^{(B O+(\ln (D B H) * B 1))}$ & 2.6417 & 0.5015 & \\
\hline Species & Ilex opaca & 1.40 & 16.50 & $B 0+\left(D B H^{*} B 1\right)+\left(D B H^{2} * B 2\right)$ & 4.1301 & 3.8555 & -0.1097 \\
\hline Species & Juglans nigra & 1.00 & 33.00 & $e^{(B O+(\ln (D B H) * B 1))}$ & 2.426 & 0.6078 & \\
\hline Species & Juniperus virginiana & 1.00 & 23.40 & $e^{(B 0+(\ln (D B H) * B 1))}$ & 2.2621 & 0.5944 & \\
\hline Species & Lagerstroemia indica & 1.00 & 10.20 & $e^{(B O+(\ln (D B H) * B 1))}$ & 2.6184 & 0.1761 & \\
\hline
\end{tabular}


Table 30.-Continued

\begin{tabular}{|c|c|c|c|c|c|c|c|}
\hline $\begin{array}{l}\text { Taxonomic } \\
\text { Class }\end{array}$ & Taxon & DBHMin & DBHMax & Model & BO & B1 & B2 \\
\hline Species & $\begin{array}{l}\text { Liquidambar } \\
\text { styraciflua }\end{array}$ & 1.00 & 23.00 & $B O+\left(D B H^{*} B 1\right)+\left(D B H^{2} * B 2\right)$ & 10.193 & 6.0733 & -0.1317 \\
\hline Species & Liriodendron tulipifera & 1.30 & 52.00 & $B 0+(\ln (D B H) * B 1)$ & 4.2684 & 27.5937 & \\
\hline Species & Magnolia grandiflora & 1.00 & 26.00 & $e^{(B O+(\ln (D B H) * B 1))}$ & 2.5784 & 0.457 & \\
\hline Species & Malus pumila & 1.20 & 22.00 & $e^{(B O+(\ln (D B H) * B 1))}$ & 2.3415 & 0.3107 & \\
\hline Species & Morus alba & 1.00 & 43.10 & $e^{(B O+(\ln (D B H) * B 1))}$ & 2.4252 & 0.4958 & \\
\hline Species & Morus rubra & 1.00 & 22.20 & $e^{(B O+(\ln (D B H) * B 1))}$ & 2.4371 & 0.4384 & \\
\hline Species & Picea abies & 2.50 & 27.10 & $B 0+(\ln (D B H) * B 1)$ & -6.4857 & 22.4012 & \\
\hline Species & Picea glauca & 1.00 & 19.70 & $e^{(B O+(\ln (D B H) * B 1))}$ & 2.1318 & 0.6375 & \\
\hline Species & Picea pungens & 1.50 & 21.70 & $e^{(B O+(\ln (D B H) * B 1))}$ & 1.7763 & 0.7273 & \\
\hline Species & Pinus elliottii & 5.00 & 25.00 & $B 0+(\ln (D B H) * B 1)$ & -19.9031 & 37.2196 & \\
\hline Species & Pinus nigra & 2.20 & 23.00 & $B O+\left(D B H^{*} B 1\right)+\left(D B H^{2} * B 2\right)$ & 7.7851 & 2.8191 & -0.0616 \\
\hline Species & Pinus radiata & 1.90 & 62.70 & $e^{\left(B O+\left(\ln (D B H)^{*} B 1\right)\right)}$ & 2.1094 & 0.5924 & \\
\hline Species & Pinus strobus & 1.00 & 37.20 & $B 0+\left(D B H^{*} B 1\right)+\left(D B H^{2} * B 2\right)$ & 3.7779 & 3.9924 & -0.0284 \\
\hline Species & Pinus taeda & 1.00 & 23.00 & $B 0+\left(D B H^{*} B 1\right)+\left(D B H^{2} * B 2\right)$ & 3.6528 & 6.7587 & -0.15 \\
\hline Species & Pinus virginiana & 1.10 & 19.00 & $e^{(B O+(\ln (D B H) * B 1))}$ & 2.0008 & 0.7644 & \\
\hline Species & Platanus hybrida & 1.00 & 45.00 & $e^{(B O+(\ln (D B H) * B 1))}$ & 2.2615 & 0.5779 & \\
\hline Species & Platanus occidentalis & 1.00 & 51.20 & $e^{(B O+(\ln (D B H) * B 1))}$ & 2.6718 & 0.5512 & \\
\hline Species & Populus balsamifera & 1.00 & 23.20 & $B 0+(\ln (D B H) * B 1)$ & 0.5398 & 23.3071 & \\
\hline Species & Populus deltoides & 1.00 & 24.00 & $B 0+\left(D B H^{*} B 1\right)+\left(D B H^{2} * B 2\right)$ & 5.0638 & 6.2902 & -0.1315 \\
\hline Species & Populus tremuloides & 1.00 & 15.80 & $B 0+\left(D B H^{*} B 1\right)$ & 6.7078 & 4.541 & \\
\hline Species & Prunus avium & 1.10 & 33.40 & $e^{\left(B O+\left(\ln (D B H)^{*} B 1\right)\right)}$ & 2.5504 & 0.3789 & \\
\hline Species & Prunus serotina & 1.00 & 24.00 & $B 0+\left(D B H^{*} B 1\right)+\left(D B H^{2} * B 2\right)$ & 6.447 & 5.5094 & -0.1154 \\
\hline Species & Prunus virginiana & 1.10 & 25.40 & $B 0+(\ln (D B H) * B 1)$ & 10.9243 & 9.0672 & \\
\hline Species & Pyrus calleryana & 1.10 & 15.60 & $B 0+\left(D B H^{*} B 1\right)$ & 10.5106 & 1.6266 & \\
\hline Species & Quercus alba & 1.00 & 30.00 & $B O+\left(D B H^{*} B 1\right)+\left(D B H^{2} * B 2\right)$ & 11.3288 & 5.2509 & -0.0868 \\
\hline Species & Quercus falcata & 1.00 & 37.00 & $e^{(B O+(\ln (D B H) * B 1))}$ & 2.7638 & 0.4974 & \\
\hline Species & Quercus nigra & 1.00 & 27.00 & $B 0+\left(D B H^{*} B 1\right)+\left(D B H^{2} * B 2\right)$ & 13.7152 & 4.1217 & -0.0753 \\
\hline Species & Quercus palustris & 1.00 & 40.10 & $e^{(B O+(\ln (D B H) * B 1))}$ & 2.4768 & 0.6079 & \\
\hline Species & Quercus phellos & 1.20 & 54.20 & $e^{(B O+(\ln (D B H) * B 1))}$ & 2.3827 & 0.6018 & \\
\hline Species & Quercus prinus & 1.80 & 41.50 & $e^{\left(B O+\left(\ln (D B H)^{*} B 1\right)\right)}$ & 2.6032 & 0.5449 & \\
\hline Species & Quercus rubra & 1.00 & 37.00 & $B 0+\left(D B H^{*} B 1\right)+\left(D B H^{2} * B 2\right)$ & 12.5945 & 4.3658 & -0.0594 \\
\hline Species & Quercus stellata & 1.00 & 28.00 & $e^{(B O+(\ln (D B H) * B 1))}$ & 2.5234 & 0.5601 & \\
\hline Species & Quercus velutina & 1.00 & 32.00 & $B 0+\left(D B H^{*} B 1\right)+\left(D B H^{2} * B 2\right)$ & 9.2622 & 4.8707 & -0.0765 \\
\hline Species & Quercus virginiana & 2.70 & 50.30 & $e^{(B O+(\ln (D B H) * B 1))}$ & 2.2789 & 0.4319 & \\
\hline Species & Rhamnus cathartica & 1.00 & 16.40 & $e^{(B O+(\ln (D B H) * B 1))}$ & 2.6402 & 0.1898 & \\
\hline Species & Rhus hirta & 1.00 & 5.00 & $B 0+\left(D B H^{*} B 1\right)+\left(D B H^{2 *} B 2\right)$ & 4.2632 & 6.9728 & -0.6906 \\
\hline Species & Robinia pseudoacacia & 1.00 & 24.00 & $B 0+\left(D B H^{*} B 1\right)+\left(D B H^{2} * B 2\right)$ & 6.9592 & 5.7702 & -0.1203 \\
\hline Species & Salix nigra & 1.20 & 25.20 & $e^{\left(B O+\left(\ln (D B H)^{*} B 1\right)\right)}$ & 2.6306 & 0.4739 & \\
\hline Species & Salix sericea & 1.00 & 29.30 & $e^{(B O+(\ln (D B H) * B 1))}$ & 2.6269 & 0.3717 & \\
\hline Species & Syringa vulgaris & 1.10 & 10.10 & $B 0+(\ln (D B H) * B 1)$ & 12.1013 & 5.2485 & \\
\hline Species & Thuja occidentalis & 1.00 & 35.60 & $B 0+\left(D B H^{*} B 1\right)$ & 5.8337 & 2.1101 & \\
\hline Species & Tilia americana & 1.00 & 27.00 & $B 0+\left(D B H^{*} B 1\right)+\left(D B H^{2} * B 2\right)$ & 9.8287 & 4.5034 & -0.0843 \\
\hline
\end{tabular}


Table 30.-Continued

\begin{tabular}{|c|c|c|c|c|c|c|c|}
\hline $\begin{array}{l}\text { Taxonomic } \\
\text { Class }\end{array}$ & Taxon & DBHMin & DBHMax & Model & BO & B1 & B2 \\
\hline Species & Tilia cordata & 2.40 & 27.00 & $B 0+(\ln (D B H) * B 1)$ & -4.0394 & 14.3333 & \\
\hline Species & Tsuga canadensis & 1.10 & 25.00 & $B 0+\left(D B H^{*} B 1\right)+\left(D B H^{2} * B 2\right)$ & 1.2021 & 5.1368 & -0.1035 \\
\hline Species & Ulmus alata & 1.00 & 24.00 & $e^{\left(B O+\left(\ln (D B H)^{*} B 1\right)\right)}$ & 2.4175 & 0.579 & \\
\hline Species & Ulmus americana & 1.00 & 38.00 & $e^{\left(B O+\left(\ln (D B H)^{*} B 1\right)\right)}$ & 2.5502 & 0.5341 & \\
\hline Species & Ulmus crassifolia & 1.00 & 17.00 & $B 0+\left(D B H^{*} B 1\right)+\left(D B H^{2} * B 2\right)$ & 7.1054 & 4.3431 & -0.0949 \\
\hline Species & Ulmus pumila & 1.00 & 36.00 & $e^{\left(B O+\left(\ln (D B H)^{*} B 1\right)\right)}$ & 2.3164 & 0.6227 & \\
\hline Species & Ulmus rubra & 1.10 & 26.00 & $B 0+\left(D B H^{*} B 1\right)+\left(D B H^{2} * B 2\right)$ & 8.3578 & 4.4572 & -0.0845 \\
\hline
\end{tabular}


Table 31.-Crown height equations

\begin{tabular}{|c|c|c|c|c|c|c|c|}
\hline $\begin{array}{l}\text { Taxonomic } \\
\text { Class }\end{array}$ & Taxon & DBH-Min & DBH-Max & Model & BO & B1 & B2 \\
\hline Order & Cornales & 1.00 & 27.20 & $B O+\left(D B H^{*} B 1\right)+\left(D B H^{2} * B 2\right)$ & 2.4896 & 2.7215 & -0.0273 \\
\hline Order & Dipsacales & 1.00 & 12.40 & $e^{\left(B O+\left(\ln (D B H)^{*} B 1\right)\right)}$ & 1.8437 & 0.3054 & \\
\hline Order & Ebenales & 1.00 & 16.10 & $e^{\left(B O+\left(\ln (D B H)^{*} B 1\right)\right)}$ & 1.2836 & 0.8609 & \\
\hline Order & Ericales & 1.00 & 7.00 & $e^{\left(B 0+\left(\ln (D B H)^{*} B 1\right)\right)}$ & 1.8445 & 0.5589 & \\
\hline Order & Euphorbiales & 1.00 & 20.00 & $B O+\left(D B H^{*} B 1\right)+\left(D B H^{2} * B 2\right)$ & 4.041 & 2.1568 & -0.0534 \\
\hline Order & Fabales & 1.00 & 46.20 & $B O+\left(D B H^{*} B 1\right)+\left(D B H^{2} * B 2\right)$ & 5.8865 & 2.1033 & -0.026 \\
\hline Order & Fagales & 1.00 & 32.00 & $B O+\left(D B H^{*} B 1\right)+\left(D B H^{2} * B 2\right)$ & 6.0957 & 3.0274 & -0.0467 \\
\hline Order & Gentianales & 1.00 & 16.50 & $B O+\left(D B H^{*} B 1\right)$ & 7.2447 & 1.775 & \\
\hline Order & Hamamelidales & 1.00 & 36.00 & $B O+\left(D B H^{*} B 1\right)+\left(D B H^{2 *} B 2\right)$ & 5.6684 & 2.8264 & -0.0398 \\
\hline Order & Juglandales & 1.00 & 29.00 & $B 0+\left(D B H^{*} B 1\right)+\left(D B H^{2 *} B 2\right)$ & 4.3297 & 3.3204 & -0.0576 \\
\hline Order & Laurales & 1.00 & 34.10 & $e^{(B O+(\ln (D B H) * B 1))}$ & 1.6941 & 0.5866 & \\
\hline Order & Magnoliales & 1.00 & 52.00 & $e^{(B 0+(\ln (D B H) * B 1))}$ & 2.1285 & 0.5819 & \\
\hline Order & Malvales & 1.00 & 40.00 & $B 0+\left(D B H^{*} B 1\right)+\left(D B H^{2} * B 2\right)$ & 6.0404 & 1.8306 & -0.0177 \\
\hline Order & Myrtales & 1.00 & 101.60 & $e^{\left(B O+\left(\ln (D B H)^{*} B 1\right)\right)}$ & 0.8289 & 1.0357 & \\
\hline Order & Pinales & 1.30 & 31.00 & $B O+\left(D B H^{*} B 1\right)+\left(D B H^{2} * B 2\right)$ & 3.8501 & 2.5431 & -0.0416 \\
\hline Order & Rhamnales & 1.00 & 16.40 & $e^{\left(B O+\left(\ln (D B H)^{*} B 1\right)\right)}$ & 1.6555 & 0.2883 & \\
\hline Order & Rosales & 1.00 & 28.00 & $B 0+\left(D B H^{*} B 1\right)+\left(D B H^{2} * B 2\right)$ & 4.2315 & 2.1881 & -0.0391 \\
\hline Order & Salicales & 1.00 & 29.00 & $B O+\left(D B H^{*} B 1\right)+\left(D B H^{2} * B 2\right)$ & 3.2686 & 3.101 & -0.0535 \\
\hline Order & Sapindales & 1.00 & 70.90 & $B 0+(\ln (D B H) * B 1)$ & 3.1398 & 11.1885 & \\
\hline Order & Scrophulariales & 1.00 & 27.00 & $B O+\left(D B H^{*} B 1\right)+\left(D B H^{2} * B 2\right)$ & 4.9344 & 2.6714 & -0.0488 \\
\hline Order & Urticales & 1.00 & 27.00 & $B 0+\left(D B H^{*} B 1\right)+\left(D B H^{2} * B 2\right)$ & 4.1929 & 2.7889 & -0.0523 \\
\hline Family & Aceraceae & 1.00 & 70.90 & $e^{(B O+(\ln (D B H) * B 1))}$ & 1.9873 & 0.6217 & \\
\hline Family & Anacardiaceae & 1.00 & 9.80 & $e^{(B O+(\ln (D B H) * B 1))}$ & 1.0007 & 0.2096 & \\
\hline Family & Betulaceae & 1.00 & 30.00 & $B 0+(\ln (D B H) * B 1)$ & 6.6649 & 8.3355 & \\
\hline Family & Bignoniaceae & 1.00 & 35.50 & $e^{(B O+(\ln (D B H) * B 1))}$ & 1.6777 & 0.6401 & \\
\hline Family & Cornaceae & 1.00 & 21.30 & $e^{\left(B O+\left(\ln (D B H)^{*} B 1\right)\right)}$ & 1.6482 & 0.5246 & \\
\hline Family & Cupressaceae & 1.00 & 28.00 & $B O+\left(D B H^{*} B 1\right)+\left(D B H^{2} * B 2\right)$ & 3.2638 & 2.4467 & -0.0432 \\
\hline Family & Euphorbiaceae & 1.00 & 29.30 & $B 0+\left(D B H^{*} B 1\right)+\left(D B H^{2} * B 2\right)$ & 3.9912 & 2.1772 & -0.0543 \\
\hline Family & Fagaceae & 1.00 & 32.00 & $B 0+\left(D B H^{*} B 1\right)+\left(D B H^{2} * B 2\right)$ & 6.2166 & 3.0477 & -0.0471 \\
\hline Family & Hamamelidaceae & 1.00 & 27.00 & $B 0+\left(D B H^{*} B 1\right)+\left(D B H^{2} * B 2\right)$ & 4.7545 & 3.1914 & -0.0588 \\
\hline Family & Juglandaceae & 1.00 & 29.00 & $B O+\left(D B H^{*} B 1\right)+\left(D B H^{2} * B 2\right)$ & 4.3297 & 3.3204 & -0.0576 \\
\hline Family & Lythraceae & 1.00 & 13.00 & $e^{\left(B O+\left(\ln (D B H)^{*} B 1\right)\right)}$ & 1.768 & 0.5216 & \\
\hline Family & Magnoliaceae & 1.00 & 52.00 & $e^{(B O+(\ln (D B H) * B 1))}$ & 2.1336 & 0.5797 & \\
\hline Family & Moraceae & 1.00 & 30.00 & $B 0+\left(D B H^{*} B 1\right)+\left(D B H^{2} * B 2\right)$ & 4.7656 & 2.3386 & -0.0389 \\
\hline Family & Myrtaceae & 2.00 & 101.60 & $e^{(B O+(\ln (D B H) * B 1))}$ & 0.5289 & 1.1629 & \\
\hline Family & Nyssaceae & 1.00 & 27.20 & $B 0+\left(D B H^{*} B 1\right)+\left(D B H^{2} * B 2\right)$ & 1.4715 & 4.0894 & -0.0836 \\
\hline Family & Oleaceae & 1.00 & 27.00 & $B 0+\left(D B H^{*} B 1\right)+\left(D B H^{2} * B 2\right)$ & 4.8348 & 2.7724 & -0.052 \\
\hline Family & Pinaceae & 1.30 & 33.00 & $B 0+\left(D B H^{*} B 1\right)+\left(D B H^{2} * B 2\right)$ & 4.6229 & 2.4388 & -0.0374 \\
\hline Family & Platanaceae & 1.00 & 51.20 & $B O+\left(D B H^{*} B 1\right)+\left(D B H^{2} * B 2\right)$ & 6.0828 & 2.2526 & -0.0187 \\
\hline Family & Rhamnaceae & 1.00 & 16.40 & $e^{(B O+(\ln (D B H) * B 1))}$ & 1.6577 & 0.2885 & \\
\hline Family & Rosaceae & 1.00 & 28.00 & $B 0+\left(D B H^{*} B 1\right)+\left(D B H^{2} * B 2\right)$ & 4.2315 & 2.1904 & -0.0393 \\
\hline Family & Rutaceae & 1.00 & 17.00 & $e^{\left(B O+\left(\ln (D B H)^{*} B 1\right)\right)}$ & 1.8781 & 0.3425 & \\
\hline
\end{tabular}


Table 31.-Continued

\begin{tabular}{|c|c|c|c|c|c|c|c|}
\hline $\begin{array}{l}\text { Taxonomic } \\
\text { Class }\end{array}$ & Taxon & DBH-Min & DBH-Max & Model & BO & B1 & B2 \\
\hline Family & Salicaceae & 1.00 & 29.00 & $B O+\left(D B H^{*} B 1\right)+\left(D B H^{2} * B 2\right)$ & 3.2686 & 3.101 & -0.0535 \\
\hline Family & Scrophulariaceae & 1.00 & 23.00 & $e^{\left(B 0+\left(\ln (D B H)^{*} B 1\right)\right)}$ & 2.2526 & 0.2133 & \\
\hline Family & Simaroubaceae & 1.00 & 21.00 & $B O+\left(D B H^{*} B 1\right)+\left(D B H^{2} * B 2\right)$ & 3.0956 & 2.6435 & -0.0636 \\
\hline Family & Tiliaceae & 1.00 & 40.00 & $B 0+\left(D B H^{*} B 1\right)+\left(D B H^{2} * B 2\right)$ & 6.2999 & 1.822 & -0.0177 \\
\hline Family & Ulmaceae & 1.00 & 25.00 & $B O+\left(D B H^{*} B 1\right)+\left(D B H^{2} * B 2\right)$ & 3.9563 & 2.9598 & -0.0582 \\
\hline Genus & Betula & 1.00 & 30.00 & $e^{(B O+(\ln (D B H) * B 1))}$ & 2.3223 & 0.4633 & \\
\hline Genus & Carpinus & 1.00 & 15.20 & $e^{(B O+(\ln (D B H) * B 1))}$ & 2.1483 & 0.4204 & \\
\hline Genus & Carya & 1.00 & 27.00 & $B 0+\left(D B H^{*} B 1\right)+\left(D B H^{2 *} B 2\right)$ & 4.036 & 3.5895 & -0.067 \\
\hline Genus & Catalpa & 1.00 & 35.50 & $e^{(B O+(\ln (D B H) * B 1))}$ & 1.6694 & 0.6537 & \\
\hline Genus & Celtis & 1.00 & 25.10 & $e^{(B O+(\ln (D B H) * B 1))}$ & 1.7516 & 0.6714 & \\
\hline Genus & Crataegus & 1.00 & 19.70 & $e^{(B O+(\ln (D B H) * B 1))}$ & 1.6385 & 0.407 & \\
\hline Genus & Cupressus & 2.40 & 61.00 & $B 0+(\ln (D B H) * B 1)$ & 8.473 & 4.8715 & \\
\hline Genus & Eucalyptus & 2.00 & 101.60 & $e^{(B O+(\ln (D B H) * B 1))}$ & 0.4193 & 1.221 & \\
\hline Genus & Fagus & 1.00 & 27.00 & $B O+\left(D B H^{*} B 1\right)+\left(D B H^{2} * B 2\right)$ & 3.8856 & 4.9363 & -0.0931 \\
\hline Genus & Fraxinus & 1.00 & 25.00 & $B 0+\left(D B H^{*} B 1\right)+\left(D B H^{2} * B 2\right)$ & 5.211 & 2.872 & -0.0567 \\
\hline Genus & Gleditsia & 1.30 & 39.00 & $B 0+\left(D B H^{*} B 1\right)+\left(D B H^{2 *} B 2\right)$ & 6.98 & 1.7483 & -0.0227 \\
\hline Genus & Juglans & 1.00 & 33.00 & $B 0+\left(D B H^{*} B 1\right)+\left(D B H^{2} * B 2\right)$ & 3.6877 & 2.6559 & -0.0291 \\
\hline Genus & Juniperus & 1.00 & 20.00 & $B 0+\left(D B H^{*} B 1\right)+\left(D B H^{2} * B 2\right)$ & 0.9801 & 3.8464 & -0.096 \\
\hline Genus & Liquidambar & 1.00 & 27.00 & $B O+\left(D B H^{*} B 1\right)+\left(D B H^{2} * B 2\right)$ & 4.8531 & 3.1701 & -0.058 \\
\hline Genus & Liriodendron & 1.00 & 52.00 & $e^{(B 0+(\ln (D B H) * B 1))}$ & 2.1066 & 0.6022 & \\
\hline Genus & Magnolia & 1.00 & 50.30 & $e^{(B 0+(\ln (D B H) * B 1))}$ & 2.2992 & 0.4318 & \\
\hline Genus & Malus & 1.00 & 29.00 & $e^{(B O+(\ln (D B H) * B 1))}$ & 2.0303 & 0.304 & \\
\hline Genus & Morus & 1.00 & 24.00 & $B O+\left(D B H^{*} B 1\right)+\left(D B H^{2} * B 2\right)$ & 4.628 & 2.4977 & -0.053 \\
\hline Genus & Ostrya & 1.00 & 13.80 & $e^{(B O+(\ln (D B H) * B 1))}$ & 1.9715 & 0.5583 & \\
\hline Genus & Phellodendron & 1.00 & 17.00 & $e^{(B O+(\ln (D B H) * B 1))}$ & 1.8867 & 0.3916 & \\
\hline Genus & Picea & 1.00 & 27.10 & $B 0+\left(D B H^{*} B 1\right)+\left(D B H^{2} * B 2\right)$ & 4.1574 & 3.1523 & -0.0524 \\
\hline Genus & Pinus & 1.00 & 34.00 & $B O+\left(D B H^{*} B 1\right)+\left(D B H^{2} * B 2\right)$ & 4.7157 & 2.216 & -0.0326 \\
\hline Genus & Populus & 1.00 & 36.00 & $B 0+\left(D B H^{*} B 1\right)+\left(D B H^{2} * B 2\right)$ & 3.3049 & 3.0906 & -0.0431 \\
\hline Genus & Prunus & 1.00 & 26.00 & $B 0+\left(D B H^{*} B 1\right)+\left(D B H^{2} * B 2\right)$ & 3.9189 & 2.544 & -0.0486 \\
\hline Genus & Pyrus & 1.00 & 19.40 & $B 0+\left(D B H^{*} B 1\right)$ & 7.5071 & 1.1779 & \\
\hline Genus & Quercus & 1.00 & 33.00 & $B O+\left(D B H^{*} B 1\right)+\left(D B H^{2} * B 2\right)$ & 5.4554 & 2.9539 & -0.0445 \\
\hline Genus & Rhus & 1.00 & 9.80 & $e^{\left(B O+\left(\ln (D B H)^{*} B 1\right)\right)}$ & 1.0038 & 0.1913 & \\
\hline Genus & Robinia & 1.00 & 35.00 & $B O+\left(D B H^{*} B 1\right)+\left(D B H^{2} * B 2\right)$ & 5.9049 & 2.1987 & -0.0235 \\
\hline Genus & Salix & 1.00 & 41.30 & $e^{\left(B O+\left(\ln (D B H)^{*} B 1\right)\right)}$ & 1.6529 & 0.6577 & \\
\hline Genus & Sapium & 1.00 & 20.00 & $B O+\left(D B H^{*} B 1\right)+\left(D B H^{2} * B 2\right)$ & 3.9912 & 2.1772 & -0.0543 \\
\hline Genus & Sassafras & 1.00 & 34.10 & $e^{\left(B O+\left(\ln (D B H)^{*} B 1\right)\right)}$ & 1.5928 & 0.6384 & \\
\hline Genus & Syringa & 1.00 & 10.10 & $B 0+(D B H * B 1)$ & 3.5307 & 1.0628 & \\
\hline Genus & Thuja & 1.00 & 35.60 & $B 0+\left(D B H^{*} B 1\right)$ & 4.7348 & 1.5925 & \\
\hline Genus & Tsuga & 1.10 & 36.00 & $B O+\left(D B H^{*} B 1\right)+\left(D B H^{2} * B 2\right)$ & 2.9814 & 2.552 & -0.0218 \\
\hline Genus & Ulmus & 1.00 & 27.00 & $B 0+\left(D B H^{*} B 1\right)+\left(D B H^{2} * B 2\right)$ & 4.0399 & 2.9476 & -0.055 \\
\hline Genus & Viburnum & 1.00 & 9.50 & $e^{(B O+(\ln (D B H) * B 1))}$ & 1.8853 & 0.264 & \\
\hline Species & Acer negundo & 1.00 & 21.00 & $B O+\left(D B H^{*} B 1\right)+\left(D B H^{2}{ }^{*} B 2\right)$ & 4.4778 & 3.1358 & -0.0742 \\
\hline
\end{tabular}


Table 31.-Continued

\begin{tabular}{|c|c|c|c|c|c|c|c|}
\hline $\begin{array}{l}\text { Taxonomic } \\
\text { Class }\end{array}$ & Taxon & DBH-Min & DBH-Max & Model & BO & B1 & B2 \\
\hline Species & Acer palmatum & 1.00 & 23.20 & $B 0+\left(D B H^{*} B 1\right)$ & 4.8807 & 1.1865 & \\
\hline Species & Acer platanoides & 1.00 & 37.60 & $e^{\left(B O+\left(\ln (D B H)^{*} B 1\right)\right)}$ & 2.0734 & 0.5317 & \\
\hline Species & Acer pseudoplatanus & 1.00 & 53.20 & $e^{(B 0+(\ln (D B H) * B 1))}$ & 2.1447 & 0.5258 & \\
\hline Species & Acer rubrum & 1.00 & 45.00 & $e^{(B O+(\ln (D B H) * B 1))}$ & 1.9422 & 0.6517 & \\
\hline Species & Acer saccharinum & 1.00 & 70.90 & $e^{(B O+(\ln (D B H) * B 1))}$ & 2.0482 & 0.5967 & \\
\hline Species & Acer saccharum & 1.00 & 40.00 & $B 0+(\ln (D B H) * B 1)$ & 4.3008 & 13.1534 & \\
\hline Species & Betula papyrifera & 1.00 & 21.30 & $B 0+(\ln (D B H) * B 1)$ & 8.6195 & 8.8565 & \\
\hline Species & Betula pendula & 1.00 & 30.00 & $B 0+(\ln (D B H) * B 1)$ & 13.2422 & 9.4508 & \\
\hline Species & Betula populifolia & 1.00 & 12.20 & $e^{\left(B O+\left(\ln (D B H)^{*} B 1\right)\right)}$ & 2.331 & 0.4096 & \\
\hline Species & Carpinus caroliniana & 1.00 & 7.00 & $B O+\left(D B H^{*} B 1\right)+\left(D B H^{2} * B 2\right)$ & 3.9616 & 4.5169 & -0.3409 \\
\hline Species & Carya alba & 1.00 & 22.00 & $B O+\left(D B H^{*} B 1\right)+\left(D B H^{2} * B 2\right)$ & 3.4081 & 4.5112 & -0.1021 \\
\hline Species & Carya cordiformis & 1.00 & 33.00 & $e^{(B O+(\ln (D B H) * B 1))}$ & 1.8466 & 0.7115 & \\
\hline Species & Carya glabra & 1.00 & 23.90 & $e^{\left(B O+\left(\ln (D B H)^{*} B 1\right)\right)}$ & 1.9634 & 0.7264 & \\
\hline Species & Carya illinoinensis & 1.50 & 35.00 & $e^{(B O+(\ln (D B H) * B 1))}$ & 2.2766 & 0.4262 & \\
\hline Species & Carya ovata & 1.00 & 25.20 & $B 0+\left(D B H^{*} B 1\right)$ & 5.8375 & 2.4594 & \\
\hline Species & Catalpa speciosa & 1.00 & 35.50 & $e^{(B 0+(\ln (D B H) * B 1))}$ & 1.6645 & 0.6539 & \\
\hline Species & Celtis laevigata & 1.30 & 25.00 & $e^{(B O+(\ln (D B H) * B 1))}$ & 2.158 & 0.4732 & \\
\hline Species & Celtis occidentalis & 1.00 & 25.10 & $e^{\left(B O+\left(\ln (D B H)^{*} B 1\right)\right)}$ & 1.5917 & 0.7817 & \\
\hline Species & Cornus florida & 1.00 & 17.60 & $e^{(B O+(\ln (D B H) * B 1))}$ & 1.6619 & 0.5154 & \\
\hline Species & Eucalyptus globulus & 2.00 & 101.60 & $e^{(B 0+(\ln (D B H) * B 1))}$ & 0.3438 & 1.2775 & \\
\hline Species & Fagus grandifolia & 1.00 & 26.00 & $B 0+\left(D B H^{*} B 1\right)+\left(D B H^{2} * B 2\right)$ & 3.858 & 4.9592 & -0.0939 \\
\hline Species & Fraxinus americana & 1.00 & 26.00 & $B 0+\left(D B H^{*} B 1\right)+\left(D B H^{2} * B 2\right)$ & 4.9291 & 2.9204 & -0.0554 \\
\hline Species & Fraxinus pennsylvanica & 1.00 & 40.00 & $e^{(B O+(\ln (D B H) * B 1))}$ & 2.0069 & 0.567 & \\
\hline Species & Ilex opaca & 1.40 & 16.50 & $B 0+\left(D B H^{*} B 1\right)$ & 4.6572 & 2.3151 & \\
\hline Species & Juglans nigra & 1.00 & 33.00 & $B 0+\left(D B H^{*} B 1\right)+\left(D B H^{2} * B 2\right)$ & 2.9058 & 2.8986 & -0.038 \\
\hline Species & Juniperus virginiana & 1.00 & 23.40 & $e^{(B 0+(\ln (D B H) * B 1))}$ & 2.0926 & 0.5665 & \\
\hline Species & Lagerstroemia indica & 1.00 & 10.20 & $B 0+\left(D B H^{*} B 1\right)$ & 4.8082 & 1.6692 & \\
\hline Species & Liquidambar styraciflua & 1.00 & 27.00 & $B 0+\left(D B H^{*} B 1\right)+\left(D B H^{2} * B 2\right)$ & 4.9091 & 3.1597 & -0.0576 \\
\hline Species & Liriodendron tulipifera & 1.30 & 52.00 & $e^{\left(B O+\left(\ln (D B H)^{*} B 1\right)\right)}$ & 2.1078 & 0.6018 & \\
\hline Species & Magnolia grandiflora & 1.00 & 26.00 & $e^{(B 0+(\ln (D B H) * B 1))}$ & 2.3096 & 0.496 & \\
\hline Species & Malus pumila & 1.20 & 22.00 & $e^{(B O+(\ln (D B H) * B 1))}$ & 2.1383 & 0.2531 & \\
\hline Species & Morus alba & 1.00 & 24.00 & $B 0+\left(D B H^{*} B 1\right)+\left(D B H^{2} * B 2\right)$ & 5.0542 & 2.4817 & -0.0524 \\
\hline Species & Morus rubra & 1.00 & 22.20 & $e^{\left(B O+\left(\ln (D B H)^{*} B 1\right)\right)}$ & 1.9783 & 0.5286 & \\
\hline Species & Picea abies & 2.50 & 27.10 & $B 0+(D B H * B 1)$ & 8.2522 & 2.0189 & \\
\hline Species & Picea glauca & 1.00 & 19.70 & $e^{(B O+(\ln (D B H) * B 1))}$ & 2.0261 & 0.6099 & \\
\hline Species & Picea pungens & 1.50 & 21.70 & $B 0+\left(D B H^{*} B 1\right)+\left(D B H^{2} * B 2\right)$ & 3.4727 & 2.8321 & -0.0441 \\
\hline Species & Pinus elliottii & 5.00 & 25.00 & $B 0+\left(D B H^{*} B 1\right)$ & 9.5228 & 1.3195 & \\
\hline Species & Pinus nigra & 2.20 & 24.00 & $B O+\left(D B H^{*} B 1\right)+\left(D B H^{2} * B 2\right)$ & 7.2858 & 1.7886 & -0.0379 \\
\hline Species & Pinus radiata & 1.90 & 62.70 & $e^{(B O+(\ln (D B H) * B 1))}$ & 2.055 & 0.4619 & \\
\hline Species & Pinus strobus & 1.00 & 31.00 & $B 0+\left(D B H^{*} B 1\right)+\left(D B H^{2} * B 2\right)$ & 3.2668 & 3.0301 & -0.0485 \\
\hline Species & Pinus taeda & 1.00 & 34.00 & $e^{(B O+(\ln (D B H) * B 1))}$ & 1.6624 & 0.6361 & \\
\hline Species & Pinus virginiana & 1.10 & 19.00 & $e^{(B 0+(\ln (D B H) * B 1))}$ & 1.4992 & 0.6753 & \\
\hline
\end{tabular}


Table 31.-Continued

\begin{tabular}{|c|c|c|c|c|c|c|c|}
\hline $\begin{array}{l}\text { Taxonomic } \\
\text { Class }\end{array}$ & Taxon & DBH-Min & DBH-Max & Model & BO & B1 & B2 \\
\hline Species & Platanus hybrida & 1.00 & 45.00 & $e^{(B O+(\ln (D B H) * B 1))}$ & 1.6125 & 0.6897 & \\
\hline Species & Platanus occidentalis & 1.00 & 51.20 & $B 0+\left(D B H^{*} B 1\right)+\left(D B H^{2} * B 2\right)$ & 4.9825 & 2.5967 & -0.0176 \\
\hline Species & Populus balsamifera & 1.00 & 23.20 & $B 0+\left(D B H^{*} B 1\right)+\left(D B H^{2} * B 2\right)$ & 6.8646 & 1.0936 & 0.133 \\
\hline Species & Populus deltoides & 1.00 & 41.80 & $e^{(B O+(\ln (D B H) * B 1))}$ & 2.2951 & 0.4959 & \\
\hline Species & Populus tremuloides & 1.00 & 9.00 & $B 0+\left(D B H^{*} B 1\right)+\left(D B H^{2} * B 2\right)$ & 1.4702 & 4.3657 & -0.2558 \\
\hline Species & Prunus avium & 1.10 & 33.40 & $e^{(B O+(\ln (D B H) * B 1))}$ & 2.0145 & 0.4617 & \\
\hline Species & Prunus serotina & 1.00 & 27.00 & $B 0+\left(D B H^{*} B 1\right)+\left(D B H^{2} * B 2\right)$ & 3.8327 & 2.7042 & -0.0492 \\
\hline Species & Prunus virginiana & 1.10 & 25.40 & $B 0+(\ln (D B H) * B 1)$ & 5.6824 & 7.617 & \\
\hline Species & Pyrus calleryana & 1.10 & 15.60 & $B 0+\left(D B H^{*} B 1\right)$ & 7.677 & 1.18 & \\
\hline Species & Quercus alba & 1.00 & 32.00 & $B 0+\left(D B H^{*} B 1\right)+\left(D B H^{2} * B 2\right)$ & 5.8887 & 3.1019 & -0.0483 \\
\hline Species & Quercus falcata & 1.00 & 33.00 & $B 0+\left(D B H^{*} B 1\right)+\left(D B H^{2} * B 2\right)$ & 4.4512 & 3.0397 & -0.0464 \\
\hline Species & Quercus nigra & 1.00 & 27.00 & $B O+\left(D B H^{*} B 1\right)+\left(D B H^{2} * B 2\right)$ & 4.2006 & 3.2994 & -0.0609 \\
\hline Species & Quercus palustris & 1.00 & 40.10 & $e^{\left(B O+\left(\ln (D B H)^{*} B 1\right)\right)}$ & 1.8501 & 0.6992 & \\
\hline Species & Quercus phellos & 1.20 & 54.20 & $B 0+\left(D B H^{*} B 1\right)$ & 9.6871 & 1.9065 & \\
\hline Species & Quercus prinus & 1.80 & 41.50 & $e^{(B O+(\ln (D B H) * B 1))}$ & 2.2264 & 0.4799 & \\
\hline Species & Quercus rubra & 1.00 & 67.00 & $B 0+(\ln (D B H) * B 1)$ & 0.4641 & 13.7235 & \\
\hline Species & Quercus stellata & 1.00 & 28.00 & $e^{\left(B O+\left(\ln (D B H)^{*} B 1\right)\right)}$ & 1.5045 & 0.7604 & \\
\hline Species & Quercus velutina & 1.50 & 54.60 & $B 0+(\ln (D B H) * B 1)$ & -1.6038 & 14.3242 & \\
\hline Species & Quercus virginiana & 2.70 & 50.30 & $e^{(B O+(\ln (D B H) * B 1))}$ & 1.8411 & 0.4913 & \\
\hline Species & Rhamnus cathartica & 1.00 & 16.40 & $e^{(B O+(\ln (D B H) * B 1))}$ & 1.6648 & 0.2501 & \\
\hline Species & Robinia pseudoacacia & 1.00 & 35.00 & $B 0+\left(D B H^{*} B 1\right)+\left(D B H^{2} * B 2\right)$ & 5.9067 & 2.2067 & -0.0245 \\
\hline Species & Salix nigra & 1.20 & 25.20 & $e^{\left(B 0+\left(\ln (D B H)^{*} B 1\right)\right)}$ & 1.9764 & 0.5817 & \\
\hline Species & Salix sericea & 1.00 & 20.00 & $B 0+\left(D B H^{*} B 1\right)+\left(D B H^{2} * B 2\right)$ & 3.9912 & 2.1772 & -0.0543 \\
\hline Species & Syringa vulgaris & 1.10 & 10.10 & $B 0+\left(D B H^{*} B 1\right)$ & 2.9685 & 1.14 & \\
\hline Species & Thuja occidentalis & 1.00 & 35.60 & $B 0+(D B H * B 1)$ & 4.8903 & 1.4952 & \\
\hline Species & Tilia americana & 1.00 & 40.00 & $B 0+\left(D B H^{*} B 1\right)+\left(D B H^{2} * B 2\right)$ & 5.3963 & 2.3592 & -0.0274 \\
\hline Species & Tilia cordata & 2.40 & 27.00 & $e^{(B O+(\ln (D B H) * B 1))}$ & 1.4554 & 0.6788 & \\
\hline Species & Tsuga canadensis & 1.10 & 36.00 & $B 0+\left(D B H^{*} B 1\right)+\left(D B H^{2} * B 2\right)$ & 3.0025 & 2.5558 & -0.0221 \\
\hline Species & Ulmus alata & 1.00 & 24.00 & $B 0+\left(D B H^{*} B 1\right)$ & 4.74 & 2.2668 & \\
\hline Species & Ulmus americana & 1.00 & 38.00 & $e^{\left(B O+\left(\ln (D B H)^{*} B 1\right)\right)}$ & 1.8999 & 0.6114 & \\
\hline Species & Ulmus crassifolia & 1.00 & 17.00 & $e^{(B O+(\ln (D B H) * B 1))}$ & 1.7337 & 0.7143 & \\
\hline Species & Ulmus pumila & 1.00 & 36.00 & $e^{\left(B O+\left(\ln (D B H)^{*} B 1\right)\right)}$ & 1.7744 & 0.6095 & \\
\hline Species & Ulmus rubra & 1.10 & 28.00 & $B O+\left(D B H^{*} B 1\right)+\left(D B H^{2} * B 2\right)$ & 3.1371 & 3.1945 & -0.0578 \\
\hline
\end{tabular}


Table 32.-Crown width equations

\begin{tabular}{|c|c|c|c|c|c|c|c|}
\hline $\begin{array}{l}\text { Taxonomic } \\
\text { Class }\end{array}$ & Taxon & $\begin{array}{l}\text { DBH- } \\
\text { Min }\end{array}$ & $\begin{array}{l}\text { DBH- } \\
\text { Max }\end{array}$ & Model & B0 & B1 & B2 \\
\hline Order & Cornales & 1.00 & 27.20 & $\mathrm{~B} 0+(\mathrm{DBH} * \mathrm{~B} 1)+\left(\mathrm{DBH}^{2} * \mathrm{~B} 2\right)$ & 5.8018 & 2.4278 & -0.0473 \\
\hline Order & Dipsacales & 1.00 & 12.40 & $\mathrm{~B} 0+(\mathrm{DBH} * \mathrm{~B} 1)$ & 9.104 & 0.6597 & \\
\hline Order & Ebenales & 1.00 & 16.10 & $\mathrm{e}^{(\mathrm{B} 0+(\ln (\mathrm{DBH}) * \mathrm{~B} 1))}$ & 1.7794 & 0.6205 & \\
\hline Order & Ericales & 1.00 & 7.00 & $\mathrm{e}^{(\mathrm{BO}+(\ln (\mathrm{DBH}) * \mathrm{~B} 1))}$ & 1.9878 & 0.4638 & \\
\hline Order & Euphorbiales & 1.00 & 29.30 & $\mathrm{e}^{(\mathrm{B} 0+(\ln (\mathrm{DBH}) * \mathrm{~B} 1))}$ & 1.5739 & 0.6114 & \\
\hline Order & Fabales & 1.00 & 29.00 & $\mathrm{~B} 0+(\mathrm{DBH} * \mathrm{~B} 1)+\left(\mathrm{DBH}^{2} * \mathrm{~B} 2\right)$ & 6.5789 & 2.0282 & -0.0344 \\
\hline Order & Fagales & 1.00 & 67.00 & $\mathrm{~B} 0+(\mathrm{DBH} * \mathrm{~B} 1)+\left(\mathrm{DBH}^{2} * \mathrm{~B} 2\right)$ & 7.1598 & 1.8053 & -0.013 \\
\hline Order & Gentianales & 1.00 & 16.50 & $\mathrm{e}^{\left(\mathrm{BO}+\left(\ln (\mathrm{DBH})^{*} \mathrm{~B} 1\right)\right)}$ & 1.9985 & 0.3371 & \\
\hline Order & Hamamelidales & 1.00 & 51.20 & $\mathrm{~B} 0+(\mathrm{DBH} * \mathrm{~B} 1)$ & 5.3249 & 1.5446 & \\
\hline Order & Juglandales & 1.00 & 44.00 & $\mathrm{~B} 0+(\mathrm{DBH} * \mathrm{~B} 1)+\left(\mathrm{DBH}^{2} * \mathrm{~B} 2\right)$ & 5.9877 & 2.0752 & -0.0236 \\
\hline Order & Laurales & 1.00 & 34.10 & $\mathrm{~B} 0+(\mathrm{DBH} * \mathrm{~B} 1)+\left(\mathrm{DBH}^{2} * \mathrm{~B} 2\right)$ & 5.4106 & 2.023 & -0.0303 \\
\hline Order & Magnoliales & 1.00 & 52.00 & $\mathrm{~B} 0+(\mathrm{DBH} * \mathrm{~B} 1)+\left(\mathrm{DBH}^{2} * \mathrm{~B} 2\right)$ & 5.9132 & 1.9798 & -0.019 \\
\hline Order & Malvales & 1.00 & 40.00 & $\mathrm{~B} 0+(\mathrm{DBH} * \mathrm{~B} 1)+\left(\mathrm{DBH}^{2} * \mathrm{~B} 2\right)$ & 4.76 & 1.7351 & -0.0141 \\
\hline Order & Myrtales & 1.00 & 101.60 & $\mathrm{e}^{(\mathrm{B} 0+(\ln (\mathrm{DBH}) * \mathrm{~B} 1))}$ & 0.7593 & 0.8744 & \\
\hline Order & Pinales & 1.30 & 39.00 & $\mathrm{~B} 0+(\mathrm{DBH} * \mathrm{~B} 1)+\left(\mathrm{DBH}^{2} * \mathrm{~B} 2\right)$ & 1.786 & 1.732 & -0.0221 \\
\hline Order & Rhamnales & 1.00 & 16.40 & $\mathrm{e}^{(\mathrm{B} 0+(\ln (\mathrm{DBH}) * \mathrm{~B} 1))}$ & 1.6669 & 0.5314 & \\
\hline Order & Rosales & 1.00 & 28.00 & $\mathrm{~B} 0+(\mathrm{DBH} * \mathrm{~B} 1)+\left(\mathrm{DBH}^{2} * \mathrm{~B} 2\right)$ & 5.4942 & 1.9993 & -0.0357 \\
\hline Order & Salicales & 1.00 & 41.80 & $\mathrm{~B} 0+\left(\mathrm{DBH}{ }^{*} \mathrm{~B} 1\right)$ & 2.6658 & 1.54 & \\
\hline Order & Sapindales & 1.00 & 37.00 & $\mathrm{~B} 0+(\mathrm{DBH} * \mathrm{~B} 1)+\left(\mathrm{DBH}^{2} * \mathrm{~B} 2\right)$ & 5.775 & 2.1928 & -0.0294 \\
\hline Order & Scrophulariales & 1.00 & 33.00 & $\mathrm{~B} 0+(\mathrm{DBH} * \mathrm{~B} 1)+\left(\mathrm{DBH}^{2} * \mathrm{~B} 2\right)$ & 4.4295 & 2.2431 & -0.0344 \\
\hline Order & Urticales & 1.00 & 37.00 & $\mathrm{~B} 0+(\mathrm{DBH} * \mathrm{~B} 1)+\left(\mathrm{DBH}^{2} * \mathrm{~B} 2\right)$ & 5.4569 & 2.192 & -0.0296 \\
\hline Family & Aceraceae & 1.00 & 39.00 & $\mathrm{~B} 0+(\mathrm{DBH} * \mathrm{~B} 1)+\left(\mathrm{DBH}^{2} * \mathrm{~B} 2\right)$ & 6.3661 & 2.102 & -0.0267 \\
\hline Family & Anacardiaceae & 1.00 & 9.80 & $\mathrm{e}^{(\mathrm{B} 0+(\ln (\mathrm{DBH}) * \mathrm{~B} 1))}$ & 1.6627 & 0.4459 & \\
\hline Family & Arecaceae & 3.50 & 38.00 & $\mathrm{~B} 0+(\ln (\mathrm{DBH}) * \mathrm{~B} 1)$ & 0.2355 & 3.7689 & \\
\hline Family & Betulaceae & 1.00 & 30.00 & $\mathrm{~B} 0+(\mathrm{DBH} * \mathrm{~B} 1)+\left(\mathrm{DBH}^{2} * \mathrm{~B} 2\right)$ & 7.0333 & 1.9312 & -0.0309 \\
\hline Family & Bignoniaceae & 1.00 & 35.50 & $\mathrm{e}^{\left(\mathrm{B} 0+\left(\ln (\mathrm{DBH})^{*} \mathrm{~B} 1\right)\right)}$ & 2.1261 & 0.4604 & \\
\hline Family & Cornaceae & 1.00 & 21.30 & $\mathrm{e}^{(\mathrm{B} 0+(\ln (\mathrm{DBH}) * \mathrm{~B} 1))}$ & 2.0322 & 0.5071 & \\
\hline Family & Cupressaceae & 1.00 & 61.00 & $\mathrm{e}^{(\mathrm{BO}+(\ln (\mathrm{DBH}) * \mathrm{~B} 1))}$ & 1.0354 & 0.6262 & \\
\hline Family & Euphorbiaceae & 1.00 & 29.30 & $\mathrm{e}^{(\mathrm{B} 0+(\ln (\mathrm{DBH}) * \mathrm{~B} 1))}$ & 1.5732 & 0.6126 & \\
\hline Family & Fagaceae & 1.00 & 67.00 & $\mathrm{~B} 0+(\mathrm{DBH} * \mathrm{~B} 1)+\left(\mathrm{DBH}^{2} * \mathrm{~B} 2\right)$ & 7.122 & 1.8167 & -0.0132 \\
\hline Family & Hamamelidaceae & 1.00 & 40.00 & $\mathrm{~B} 0+(\mathrm{DBH} * \mathrm{~B} 1)$ & 5.5438 & 1.4528 & \\
\hline Family & Lythraceae & 1.00 & 13.00 & $\exp (B 0+(\ln (D B H) * B 1$ & 1.917 & 0.4138 & \\
\hline Family & Magnoliaceae & 1.00 & 52.00 & $\mathrm{~B} 0+(\mathrm{DBH} * \mathrm{~B} 1)+\left(\mathrm{DBH}^{2} * \mathrm{~B} 2\right)$ & 5.906 & 1.9807 & -0.0191 \\
\hline Family & Moraceae & 1.00 & 58.00 & $B 0+(\ln (D B H) * B 1)$ & 4.9539 & 8.2482 & \\
\hline Family & Myrtaceae & 2.00 & 101.60 & $\mathrm{e}^{(\mathrm{B} 0+(\ln (\mathrm{DBH}) * \mathrm{~B} 1))}$ & 0.0761 & 1.1534 & \\
\hline Family & Nyssaceae & 1.00 & 27.20 & $\mathrm{~B} 0+(\mathrm{DBH} * \mathrm{~B} 1)+\left(\mathrm{DBH}^{2} * \mathrm{~B} 2\right)$ & 5.9613 & 2.0986 & -0.0233 \\
\hline Family & Oleaceae & 1.00 & 35.00 & $\mathrm{~B} 0+(\mathrm{DBH} * \mathrm{~B} 1)+\left(\mathrm{DBH}^{2} * \mathrm{~B} 2\right)$ & 4.3871 & 2.2158 & -0.0317 \\
\hline Family & Pinaceae & 1.30 & 62.70 & $\mathrm{e}^{(\mathrm{B} 0+(\ln (\mathrm{DBH}) * \mathrm{~B} 1))}$ & 1.3208 & 0.664 & \\
\hline Family & Platanaceae & 1.00 & 51.20 & $\mathrm{~B} 0+(\mathrm{DBH} * \mathrm{~B} 1)+\left(\mathrm{DBH}^{2} * \mathrm{~B} 2\right)$ & 6.3993 & 2.0634 & -0.017 \\
\hline Family & Rhamnaceae & 1.00 & 16.40 & $\mathrm{e}^{\left(\mathrm{B} 0+\left(\ln (\mathrm{DBH})^{*} \mathrm{~B} 1\right)\right)}$ & 1.6555 & 0.5428 & \\
\hline Family & Rosaceae & 1.00 & 28.00 & $\mathrm{~B} 0+(\mathrm{DBH} * \mathrm{~B} 1)+\left(\mathrm{DBH}^{2} * \mathrm{~B} 2\right)$ & 5.4745 & 2.0111 & -0.0362 \\
\hline Family & Rutaceae & 1.00 & 17.00 & $\mathrm{e}^{\left(\mathrm{BO}+\left(\ln (\mathrm{DBH})^{*} \mathrm{~B} 1\right)\right)}$ & 2.2451 & 0.3713 & \\
\hline
\end{tabular}


Table 32.-Continued

\begin{tabular}{|c|c|c|c|c|c|c|c|}
\hline $\begin{array}{l}\text { Taxonomic } \\
\text { Class }\end{array}$ & Taxon & $\begin{array}{l}\text { DBH- } \\
\text { Min }\end{array}$ & $\begin{array}{l}\text { DBH- } \\
\text { Max }\end{array}$ & Model & B0 & B1 & B2 \\
\hline Family & Scrophulariaceae & 1.00 & 23.00 & $\mathrm{~B} 0+(\ln (\mathrm{DBH}) * \mathrm{~B} 1)$ & 4.1441 & 7.1404 & \\
\hline Family & Simaroubaceae & 1.00 & 31.00 & $\mathrm{~B} 0+\left(\mathrm{DBH}{ }^{*} \mathrm{~B} 1\right)+\left(\mathrm{DBH}^{2}{ }^{*} \mathrm{~B} 2\right)$ & 4.6508 & 2.5085 & -0.0456 \\
\hline Family & Tiliaceae & 1.00 & 40.00 & $\mathrm{~B} 0+\left(\mathrm{DBH}{ }^{*} \mathrm{~B} 1\right)+\left(\mathrm{DBH}^{2} * \mathrm{~B} 2\right)$ & 4.8669 & 1.7481 & -0.0148 \\
\hline Family & Ulmaceae & 1.00 & 38.00 & $\mathrm{~B} 0+(\mathrm{DBH} * \mathrm{~B} 1)+\left(\mathrm{DBH}^{2} * \mathrm{~B} 2\right)$ & 5.4986 & 2.0888 & -0.0227 \\
\hline Genus & Betula & 1.00 & 30.00 & $\mathrm{~B} 0+(\mathrm{DBH} * \mathrm{~B} 1)$ & 6.2408 & 1.5854 & \\
\hline Genus & Carpinus & 1.00 & 15.20 & $\mathrm{e}^{(\mathrm{BO}+(\ln (\mathrm{DBH}) * \mathrm{~B} 1))}$ & 2.303 & 0.4174 & \\
\hline Genus & Carya & 1.00 & 41.00 & $\mathrm{~B} 0+\left(\mathrm{DBH}{ }^{*} \mathrm{~B} 1\right)+\left(\mathrm{DBH}^{2} * \mathrm{~B} 2\right)$ & 6.035 & 2.0755 & -0.0255 \\
\hline Genus & Catalpa & 1.00 & 35.50 & $\mathrm{e}^{(\mathrm{B} 0+(\ln (\mathrm{DBH}) * \mathrm{~B} 1))}$ & 2.1204 & 0.4598 & \\
\hline Genus & Celtis & 1.00 & 21.00 & $\mathrm{~B} 0+(\mathrm{DBH} * \mathrm{~B} 1)+\left(\mathrm{DBH}^{2} * \mathrm{~B} 2\right)$ & 4.3952 & 2.6692 & -0.0625 \\
\hline Genus & Crataegus & 1.00 & 19.70 & $\mathrm{e}^{\left(\mathrm{BO}+\left(\ln (\mathrm{DBH})^{*} \mathrm{~B} 1\right)\right)}$ & 1.8242 & 0.4338 & \\
\hline Genus & Cupressus & 2.40 & 61.00 & $\mathrm{~B} 0+(\mathrm{DBH} * \mathrm{~B} 1)$ & 3.2457 & 0.6505 & \\
\hline Genus & Eucalyptus & 2.00 & 101.60 & $\mathrm{e}^{(\mathrm{BO}+(\ln (\mathrm{DBH}) * \mathrm{~B} 1))}$ & -0.0023 & 1.1862 & \\
\hline Genus & Fagus & 1.00 & 35.00 & $\mathrm{~B} 0+(\mathrm{DBH} * \mathrm{~B} 1)+\left(\mathrm{DBH}^{2} * \mathrm{~B} 2\right)$ & 7.7776 & 2.4516 & -0.0348 \\
\hline Genus & Fraxinus & 1.00 & 32.00 & $\mathrm{~B} 0+\left(\mathrm{DBH}{ }^{*} \mathrm{~B} 1\right)+\left(\mathrm{DBH}^{2}{ }^{*} \mathrm{~B} 2\right)$ & 4.5348 & 2.3021 & -0.0356 \\
\hline Genus & Gleditsia & 1.30 & 46.20 & $\mathrm{~B} 0+(\ln (\mathrm{DBH}) * \mathrm{~B} 1)$ & 1.3613 & 11.2361 & \\
\hline Genus & Juglans & 1.00 & 33.00 & $\mathrm{~B} 0+(\mathrm{DBH} * \mathrm{~B} 1)$ & 7.01 & 1.6792 & \\
\hline Genus & Juniperus & 1.00 & 23.40 & $\mathrm{~B} 0+(\mathrm{DBH} * \mathrm{~B} 1)+\left(\mathrm{DBH}^{2} * \mathrm{~B} 2\right)$ & 2.3613 & 1.764 & -0.0299 \\
\hline Genus & Liquidambar & 1.00 & 40.00 & $\mathrm{~B} 0+\left(\mathrm{DBH}{ }^{*} \mathrm{~B} 1\right)+\left(\mathrm{DBH}^{2}{ }^{*} \mathrm{~B} 2\right)$ & 4.9881 & 1.6033 & -0.0076 \\
\hline Genus & Liriodendron & 1.00 & 52.00 & $\mathrm{~B} 0+\left(\mathrm{DBH}{ }^{*} \mathrm{~B} 1\right)+\left(\mathrm{DBH}^{2} * \mathrm{~B} 2\right)$ & 5.5748 & 1.9963 & -0.0186 \\
\hline Genus & Magnolia & 1.00 & 50.30 & $\mathrm{e}^{\left(\mathrm{BO}+\left(\ln (\mathrm{DBH})^{*} \mathrm{~B} 1\right)\right)}$ & 2.0499 & 0.4761 & \\
\hline Genus & Malus & 1.00 & 29.00 & $\mathrm{e}^{(\mathrm{BO}+(\ln (\mathrm{DBH}) * \mathrm{~B} 1))}$ & 1.9915 & 0.4699 & \\
\hline Genus & Morus & 1.00 & 43.10 & $\mathrm{~B} 0+(\ln (\mathrm{DBH}) * \mathrm{~B} 1)$ & 5.0899 & 8.2704 & \\
\hline Genus & Ostrya & 1.00 & 13.80 & $\mathrm{e}^{(\mathrm{B} 0+(\ln (\mathrm{DBH}) * \mathrm{~B} 1))}$ & 1.9482 & 0.5244 & \\
\hline Genus & Phellodendron & 1.00 & 17.00 & $\mathrm{e}^{(\mathrm{B} 0+(\ln (\mathrm{DBH}) * \mathrm{~B} 1))}$ & 2.2625 & 0.4344 & \\
\hline Genus & Picea & 1.00 & 27.10 & $\mathrm{~B} 0+\left(\mathrm{DBH}{ }^{*} \mathrm{~B} 1\right)+\left(\mathrm{DBH}^{2}{ }^{*} \mathrm{~B} 2\right)$ & 2.8875 & 1.4568 & -0.0125 \\
\hline Genus & Pinus & 1.00 & 62.70 & $\mathrm{e}^{(\mathrm{B} 0+(\ln (\mathrm{DBH}) * \mathrm{~B} 1))}$ & 1.3312 & 0.6651 & \\
\hline Genus & Populus & 1.00 & 41.80 & $\mathrm{~B} 0+\left(\mathrm{DBH}{ }^{*} \mathrm{~B} 1\right)$ & 2.4739 & 1.5565 & \\
\hline Genus & Prunus & 1.00 & 30.00 & $\mathrm{~B} 0+(\mathrm{DBH} * \mathrm{~B} 1)+\left(\mathrm{DBH}^{2} * \mathrm{~B} 2\right)$ & 5.9632 & 1.9593 & -0.0327 \\
\hline Genus & Pyrus & 1.00 & 19.40 & $\mathrm{~B} 0+(\mathrm{DBH} * \mathrm{~B} 1)$ & 4.1849 & 1.5245 & \\
\hline Genus & Quercus & 1.00 & 67.00 & $\mathrm{~B} 0+\left(\mathrm{DBH}{ }^{*} \mathrm{~B} 1\right)+\left(\mathrm{DBH}^{2}{ }^{*} \mathrm{~B} 2\right)$ & 5.6153 & 1.9184 & -0.0148 \\
\hline Genus & Rhus & 1.00 & 9.80 & $\mathrm{e}^{\left(\mathrm{BO}+\left(\ln (\mathrm{DBH})^{*} \mathrm{~B} 1\right)\right)}$ & 1.6641 & 0.4392 & \\
\hline Genus & Robinia & 1.00 & 27.00 & $\mathrm{~B} 0+(\mathrm{DBH} * \mathrm{~B} 1)+\left(\mathrm{DBH}^{2} * \mathrm{~B} 2\right)$ & 6.7187 & 1.9207 & -0.035 \\
\hline Genus & Salix & 1.00 & 41.30 & $\mathrm{e}^{\left(\mathrm{BO}+\left(\ln (\mathrm{DBH})^{*} \mathrm{~B} 1\right)\right)}$ & 1.6602 & 0.5908 & \\
\hline Genus & Sassafras & 1.00 & 34.10 & $\mathrm{~B} 0+\left(\mathrm{DBH}{ }^{*} \mathrm{~B} 1\right)+\left(\mathrm{DBH}^{2} * \mathrm{~B} 2\right)$ & 4.8868 & 2.1566 & -0.0339 \\
\hline Genus & Syringa & 1.00 & 10.10 & $\mathrm{e}^{(\mathrm{B} 0+(\ln (\mathrm{DBH}) * \mathrm{~B} 1))}$ & 1.5839 & 0.4015 & \\
\hline Genus & Thuja & 1.00 & 35.60 & $\mathrm{~B} 0+\left(\mathrm{DBH}{ }^{*} \mathrm{~B} 1\right)+\left(\mathrm{DBH}^{2}{ }^{*} \mathrm{~B} 2\right)$ & 1.7205 & 1.1558 & -0.019 \\
\hline Genus & Tsuga & 1.10 & 36.00 & $\mathrm{~B} 0+(\mathrm{DBH} * \mathrm{~B} 1)+\left(\mathrm{DBH}^{2} * \mathrm{~B} 2\right)$ & 2.9363 & 1.7714 & -0.0239 \\
\hline Genus & Ulmus & 1.00 & 38.00 & $\mathrm{~B} 0+\left(\mathrm{DBH}{ }^{*} \mathrm{~B} 1\right)+\left(\mathrm{DBH}^{2} * \mathrm{~B} 2\right)$ & 5.66 & 1.9969 & -0.0177 \\
\hline Genus & Viburnum & 1.00 & 9.50 & $\mathrm{~B} 0+(\mathrm{DBH} * \mathrm{~B} 1)$ & 11.0379 & 0.189 & \\
\hline Species & Acer negundo & 1.00 & 36.00 & $\mathrm{~B} 0+\left(\mathrm{DBH}{ }^{*} \mathrm{~B} 1\right)+\left(\mathrm{DBH}^{2}{ }^{*} \mathrm{~B} 2\right)$ & 5.854 & 1.9553 & -0.0275 \\
\hline Species & Acer palmatum & 1.00 & 23.20 & $\mathrm{~B} 0+(\mathrm{DBH} * \mathrm{~B} 1)$ & 6.61 & 1.7147 & \\
\hline Species & Acer platanoides & 1.00 & 37.60 & $\mathrm{~B} 0+(\mathrm{DBH} * \mathrm{~B} 1)+\left(\mathrm{DBH}^{2} * \mathrm{~B} 2\right)$ & 5.8975 & 2.1666 & -0.0274 \\
\hline
\end{tabular}


Table 32.-Continued

\begin{tabular}{|c|c|c|c|c|c|c|c|}
\hline $\begin{array}{l}\text { Taxonomic } \\
\text { Class }\end{array}$ & Taxon & $\begin{array}{l}\text { DBH- } \\
\text { Min }\end{array}$ & $\begin{array}{l}\text { DBH- } \\
\text { Max }\end{array}$ & Model & BO & B1 & B2 \\
\hline Species & Acer pseudoplatanus & 1.00 & 53.20 & $\mathrm{e}^{(\mathrm{BO}+(\ln (\mathrm{DBH}) * \mathrm{~B} 1))}$ & 1.9314 & 0.5685 & \\
\hline Species & Acer rubrum & 1.00 & 33.00 & $\mathrm{~B} 0+\left(\mathrm{DBH}{ }^{*} \mathrm{~B} 1\right)+\left(\mathrm{DBH}^{2}{ }^{*} \mathrm{~B} 2\right)$ & 6.8474 & 2.1853 & -0.0335 \\
\hline Species & Acer saccharinum & 1.00 & 70.90 & $\mathrm{e}^{(\mathrm{B} 0+(\ln (\mathrm{DBH}) * \mathrm{~B} 1))}$ & 1.9519 & 0.5629 & \\
\hline Species & Acer saccharum & 1.00 & 32.00 & $\mathrm{~B} 0+(\mathrm{DBH} * \mathrm{~B} 1)+\left(\mathrm{DBH}^{2} * \mathrm{~B} 2\right)$ & 6.4681 & 2.2287 & -0.0351 \\
\hline Species & Betula papyrifera & 1.00 & 21.30 & $\mathrm{~B} 0+(\mathrm{DBH} * \mathrm{~B} 1)+\left(\mathrm{DBH}^{2} * \mathrm{~B} 2\right)$ & 5.064 & 1.2265 & 0.0395 \\
\hline Species & Betula pendula & 1.00 & 30.00 & $\mathrm{e}^{\left(\mathrm{BO}+\left(\ln (\mathrm{DBH})^{*} \mathrm{~B} 1\right)\right)}$ & 1.5123 & 0.571 & \\
\hline Species & Betula populifolia & 1.00 & 12.20 & $\mathrm{e}^{(\mathrm{BO}+(\ln (\mathrm{DBH}) * \mathrm{~B} 1))}$ & 1.9078 & 0.5326 & \\
\hline Species & Carpinus caroliniana & 1.00 & 10.40 & $\mathrm{~B} 0+(\ln (\mathrm{DBH}) * \mathrm{~B} 1)$ & 9.0348 & 6.7159 & \\
\hline Species & Carya alba & 1.00 & 38.50 & $\mathrm{e}^{(\mathrm{BO}+(\ln (\mathrm{DBH}) * \mathrm{~B} 1))}$ & 1.9301 & 0.5499 & \\
\hline Species & Carya cordiformis & 1.00 & 33.00 & $\mathrm{e}^{(\mathrm{BO}+(\ln (\mathrm{DBH}) * \mathrm{~B} 1))}$ & 1.946 & 0.5517 & \\
\hline Species & Carya glabra & 1.00 & 23.90 & $\mathrm{e}^{(\mathrm{BO}+(\ln (\mathrm{DBH}) * \mathrm{~B} 1))}$ & 2.0043 & 0.4994 & \\
\hline Species & Carya illinoinensis & 1.50 & 35.00 & $\mathrm{e}^{(\mathrm{BO}+(\ln (\mathrm{DBH}) * \mathrm{~B} 1))}$ & 1.7231 & 0.6653 & \\
\hline Species & Carya ovata & 1.00 & 25.20 & $\mathrm{~B} 0+(\mathrm{DBH} * \mathrm{~B} 1)$ & 6.7211 & 1.6122 & \\
\hline Species & Catalpa speciosa & 1.00 & 35.50 & $\mathrm{e}^{\left(\mathrm{B} 0+\left(\ln (\mathrm{DBH})^{*} \mathrm{~B} 1\right)\right)}$ & 2.0308 & 0.5362 & \\
\hline Species & Celtis laevigata & 1.30 & 25.00 & $\mathrm{e}^{(\mathrm{BO}+(\ln (\mathrm{DBH}) * \mathrm{~B} 1))}$ & 1.7738 & 0.6299 & \\
\hline Species & Celtis occidentalis & 1.00 & 25.10 & $\mathrm{~B} 0+(\mathrm{DBH} * \mathrm{~B} 1)+\left(\mathrm{DBH}^{2} * \mathrm{~B} 2\right)$ & 5.0384 & 2.3903 & -0.0527 \\
\hline Species & Cornus florida & 1.00 & 17.60 & $\mathrm{e}^{(\mathrm{B} 0+(\ln (\mathrm{DBH}) * \mathrm{~B} 1))}$ & 2.1047 & 0.4727 & \\
\hline Species & Eucalyptus globulus & 2.00 & 101.60 & $\mathrm{e}^{(\mathrm{B} 0+(\ln (\mathrm{DBH}) * \mathrm{~B} 1))}$ & -0.0593 & 1.233 & \\
\hline Species & Fagus grandifolia & 1.00 & 36.00 & $\mathrm{~B} 0+\left(\mathrm{DBH}{ }^{*} \mathrm{~B} 1\right)+\left(\mathrm{DBH}^{2} * \mathrm{~B} 2\right)$ & 7.8252 & 2.4359 & -0.0335 \\
\hline Species & Fraxinus americana & 1.00 & 38.00 & $\mathrm{~B} 0+(\mathrm{DBH} * \mathrm{~B} 1)+\left(\mathrm{DBH}^{2} * \mathrm{~B} 2\right)$ & 4.8708 & 2.221 & -0.0295 \\
\hline Species & Fraxinus pennsylvanica & 1.00 & 40.00 & $\mathrm{e}^{(\mathrm{B} 0+(\ln (\mathrm{DBH}) * \mathrm{~B} 1))}$ & 1.759 & 0.6078 & \\
\hline Species & Ilex opaca & 1.40 & 16.50 & $\mathrm{e}^{(\mathrm{BO}+(\ln (\mathrm{DBH}) * \mathrm{~B} 1))}$ & 1.7641 & 0.4682 & \\
\hline Species & Juglans nigra & 1.00 & 33.00 & $\mathrm{e}^{(\mathrm{BO}+(\ln (\mathrm{DBH}) * \mathrm{~B} 1))}$ & 1.8653 & 0.5878 & \\
\hline Species & Juniperus virginiana & 1.00 & 23.40 & $\mathrm{~B} 0+(\mathrm{DBH} * \mathrm{~B} 1)$ & 3.6967 & 1.2866 & \\
\hline Species & Lagerstroemia indica & 1.00 & 10.20 & $\mathrm{e}^{(\mathrm{B} 0+(\ln (\mathrm{DBH}) * \mathrm{~B} 1))}$ & 1.9526 & 0.3644 & \\
\hline Species & Liquidambar styraciflua & 1.00 & 40.00 & $\mathrm{~B} 0+(\mathrm{DBH} * \mathrm{~B} 1)+\left(\mathrm{DBH}^{2} * \mathrm{~B} 2\right)$ & 5.0207 & 1.5969 & -0.0074 \\
\hline Species & Liriodendron tulipifera & 1.30 & 52.00 & $\mathrm{~B} 0+\left(\mathrm{DBH}{ }^{*} \mathrm{~B} 1\right)+\left(\mathrm{DBH}^{2} * \mathrm{~B} 2\right)$ & 5.6119 & 1.9934 & -0.0186 \\
\hline Species & Magnolia grandiflora & 1.00 & 26.00 & $\mathrm{e}^{\left(\mathrm{BO}+\left(\ln (\mathrm{DBH})^{*} \mathrm{~B} 1\right)\right)}$ & 1.9737 & 0.4751 & \\
\hline Species & Malus pumila & 1.20 & 22.00 & $\mathrm{e}^{(\mathrm{B} 0+(\ln (\mathrm{DBH}) * \mathrm{~B} 1))}$ & 2.2312 & 0.3735 & \\
\hline Species & Morus alba & 1.00 & 43.10 & $\mathrm{~B} 0+(\ln (\mathrm{DBH}) * \mathrm{~B} 1)$ & 5.5645 & 7.96 & \\
\hline Species & Morus rubra & 1.00 & 22.20 & $\mathrm{e}^{(\mathrm{B} 0+(\ln (\mathrm{DBH}) * \mathrm{~B} 1))}$ & 2.0067 & 0.5491 & \\
\hline Species & Picea abies & 2.50 & 27.10 & $\mathrm{~B} 0+(\mathrm{DBH} * \mathrm{~B} 1)$ & 5.0275 & 1.3419 & \\
\hline Species & Picea glauca & 1.00 & 19.70 & $\mathrm{e}^{(\mathrm{B} 0+(\ln (\mathrm{DBH}) * \mathrm{~B} 1))}$ & 1.3573 & 0.5622 & \\
\hline Species & Picea pungens & 1.50 & 21.70 & $\mathrm{~B} 0+(\mathrm{DBH} * \mathrm{~B} 1)+\left(\mathrm{DBH}^{2} * \mathrm{~B} 2\right)$ & 3.1772 & 1.3664 & -0.0162 \\
\hline Species & Pinus elliottii & 5.00 & 25.00 & $\mathrm{e}^{(\mathrm{B} 0+(\ln (\mathrm{DBH}) * \mathrm{~B} 1))}$ & 1.2235 & 0.7611 & \\
\hline Species & Pinus nigra & 2.20 & 24.00 & $\mathrm{~B} 0+(\mathrm{DBH} * \mathrm{~B} 1)+\left(\mathrm{DBH}^{2} * \mathrm{~B} 2\right)$ & 5.6682 & 1.6952 & -0.036 \\
\hline Species & Pinus radiata & 1.90 & 62.70 & $\mathrm{e}^{(\mathrm{B} 0+(\ln (\mathrm{DBH}) * \mathrm{~B} 1))}$ & 1.4297 & 0.5938 & \\
\hline Species & Pinus strobus & 1.00 & 37.20 & $\mathrm{~B} 0+(\mathrm{DBH} * \mathrm{~B} 1)+\left(\mathrm{DBH}^{2} * \mathrm{~B} 2\right)$ & 3.3445 & 1.5814 & -0.0142 \\
\hline Species & Pinus taeda & 1.00 & 34.00 & $\mathrm{e}^{(\mathrm{B} 0+(\ln (\mathrm{DBH}) * \mathrm{~B} 1))}$ & 1.1535 & 0.7313 & \\
\hline Species & Pinus virginiana & 1.10 & 19.00 & $\mathrm{e}^{(\mathrm{BO}+(\ln (\mathrm{DBH}) * \mathrm{~B} 1))}$ & 1.5891 & 0.6557 & \\
\hline Species & Platanus hybrida & 1.00 & 45.00 & $\mathrm{~B} 0+(\mathrm{DBH} * \mathrm{~B} 1)+\left(\mathrm{DBH}^{2} * \mathrm{~B} 2\right)$ & 3.9088 & 2.6747 & -0.0329 \\
\hline Species & Platanus occidentalis & 1.00 & 51.20 & $\mathrm{e}^{\left(\mathrm{BO}+\left(\ln (\mathrm{DBH})^{*} \mathrm{~B} 1\right)\right)}$ & 1.9074 & 0.5682 & \\
\hline
\end{tabular}


Table 32.-Continued

\begin{tabular}{|c|c|c|c|c|c|c|c|}
\hline $\begin{array}{l}\text { Taxonomic } \\
\text { Class }\end{array}$ & Taxon & $\begin{array}{l}\text { DBH- } \\
\text { Min }\end{array}$ & $\begin{array}{l}\text { DBH- } \\
\text { Max }\end{array}$ & Model & BO & B1 & B2 \\
\hline Species & Populus balsamifera & 1.00 & 23.20 & $\mathrm{~B} 0+\left(\mathrm{DBH}{ }^{*} \mathrm{~B} 1\right)$ & 4.1386 & 1.1984 & \\
\hline Species & Populus deltoides & 1.00 & 41.80 & $\mathrm{~B} 0+(\mathrm{DBH} * \mathrm{~B} 1)$ & 4.3047 & 1.6294 & \\
\hline Species & Populus tremuloides & 1.00 & 9.00 & $\mathrm{~B} 0+(\mathrm{DBH} * \mathrm{~B} 1)+\left(\mathrm{DBH}^{2} * \mathrm{~B} 2\right)$ & 1.2786 & 2.3693 & -0.1346 \\
\hline Species & Prunus avium & 1.10 & 33.40 & $\mathrm{e}^{\left(\mathrm{BO}+\left(\ln (\mathrm{DBH})^{*} \mathrm{~B} 1\right)\right)}$ & 1.9615 & 0.5056 & \\
\hline Species & Prunus serotina & 1.00 & 28.00 & $\mathrm{~B} 0+(\mathrm{DBH} * \mathrm{~B} 1)+\left(\mathrm{DBH}^{2} * \mathrm{~B} 2\right)$ & 6.1133 & 2.0116 & -0.0355 \\
\hline Species & Prunus virginiana & 1.10 & 25.40 & $\mathrm{e}^{(\mathrm{B} 0+(\ln (\mathrm{DBH}) * \mathrm{~B} 1))}$ & 1.7052 & 0.5541 & \\
\hline Species & Pyrus calleryana & 1.10 & 15.60 & $\mathrm{~B} 0+(\mathrm{DBH} * \mathrm{~B} 1)$ & 3.3114 & 1.7738 & \\
\hline Species & Quercus alba & 1.00 & 51.60 & $\mathrm{~B} 0+(\mathrm{DBH} * \mathrm{~B} 1)+\left(\mathrm{DBH}^{2} * \mathrm{~B} 2\right)$ & 5.5617 & 1.8924 & -0.0109 \\
\hline Species & Quercus falcata & 1.00 & 37.00 & $\mathrm{~B} 0+(\mathrm{DBH} * \mathrm{~B} 1)+\left(\mathrm{DBH}^{2} * \mathrm{~B} 2\right)$ & 3.2783 & 2.3124 & -0.0246 \\
\hline Species & Quercus nigra & 1.00 & 42.00 & $\mathrm{~B} 0+(\mathrm{DBH} * \mathrm{~B} 1)+\left(\mathrm{DBH}^{2} * \mathrm{~B} 2\right)$ & 4.1202 & 2.269 & -0.0263 \\
\hline Species & Quercus palustris & 1.00 & 40.10 & $\mathrm{~B} 0+(\mathrm{DBH} * \mathrm{~B} 1)+\left(\mathrm{DBH}^{2} * \mathrm{~B} 2\right)$ & 7.7679 & 1.7229 & -0.011 \\
\hline Species & Quercus phellos & 1.20 & 37.00 & $\mathrm{~B} 0+(\mathrm{DBH} * \mathrm{~B} 1)+\left(\mathrm{DBH}^{2} * \mathrm{~B} 2\right)$ & 3.8672 & 2.1683 & -0.0297 \\
\hline Species & Quercus prinus & 1.80 & 41.50 & $\mathrm{e}^{(\mathrm{B} 0+(\ln (\mathrm{DBH}) * \mathrm{~B} 1))}$ & 2.1046 & 0.4575 & \\
\hline Species & Quercus rubra & 1.00 & 67.00 & $\mathrm{~B} 0+(\mathrm{DBH} * \mathrm{~B} 1)+\left(\mathrm{DBH}^{2} * \mathrm{~B} 2\right)$ & 6.5916 & 1.7597 & -0.011 \\
\hline Species & Quercus stellata & 1.00 & 28.00 & $\mathrm{e}^{(\mathrm{BO}+(\ln (\mathrm{DBH}) * \mathrm{~B} 1))}$ & 1.1202 & 0.8338 & \\
\hline Species & Quercus velutina & 1.00 & 54.60 & $\mathrm{~B} 0+(\mathrm{DBH} * \mathrm{~B} 1)+\left(\mathrm{DBH}^{2} * \mathrm{~B} 2\right)$ & 4.7156 & 1.8432 & -0.0116 \\
\hline Species & Quercus virginiana & 2.70 & 50.30 & $\mathrm{~B} 0+(\mathrm{DBH} * \mathrm{~B} 1)+\left(\mathrm{DBH}^{2} * \mathrm{~B} 2\right)$ & 4.905 & 1.959 & -0.0166 \\
\hline Species & Rhamnus cathartica & 1.00 & 16.40 & $\mathrm{e}^{\left(\mathrm{B} 0+\left(\ln (\mathrm{DBH})^{*} \mathrm{~B} 1\right)\right)}$ & 1.6671 & 0.5227 & \\
\hline Species & Rhus hirta & 1.00 & 9.80 & $\mathrm{e}^{(\mathrm{B} 0+(\ln (\mathrm{DBH}) * \mathrm{~B} 1))}$ & 1.6611 & 0.4203 & \\
\hline Species & Robinia pseudoacacia & 1.00 & 27.00 & $\mathrm{~B} 0+(\mathrm{DBH} * \mathrm{~B} 1)+\left(\mathrm{DBH}^{2} * \mathrm{~B} 2\right)$ & 6.4707 & 2.0431 & -0.0381 \\
\hline Species & Salix nigra & 1.20 & 25.20 & $\mathrm{e}^{(\mathrm{BO}+(\ln (\mathrm{DBH}) * \mathrm{~B} 1))}$ & 1.6136 & 0.6141 & \\
\hline Species & Salix sericea & 1.00 & 29.30 & $\mathrm{e}^{(\mathrm{B} 0+(\ln (\mathrm{DBH}) * \mathrm{~B} 1))}$ & 1.5732 & 0.6126 & \\
\hline Species & Syringa vulgaris & 1.10 & 10.10 & $\mathrm{e}^{(\mathrm{B} 0+(\ln (\mathrm{DBH}) * \mathrm{~B} 1))}$ & 1.6104 & 0.454 & \\
\hline Species & Thuja occidentalis & 1.00 & 35.60 & $\mathrm{~B} 0+(\mathrm{DBH} * \mathrm{~B} 1)+\left(\mathrm{DBH}^{2} * \mathrm{~B} 2\right)$ & 1.8741 & 1.0552 & -0.0141 \\
\hline Species & Tilia americana & 1.00 & 40.00 & $\mathrm{~B} 0+(\mathrm{DBH} * \mathrm{~B} 1)+\left(\mathrm{DBH}^{2} * \mathrm{~B} 2\right)$ & 5.2194 & 1.8045 & -0.0195 \\
\hline Species & Tilia cordata & 2.40 & 27.00 & $\mathrm{~B} 0+(\ln (\mathrm{DBH}) * \mathrm{~B} 1)$ & -11.1093 & 14.6509 & \\
\hline Species & Tsuga canadensis & 1.10 & 36.00 & $\mathrm{~B} 0+(\mathrm{DBH} * \mathrm{~B} 1)+\left(\mathrm{DBH}^{2} * \mathrm{~B} 2\right)$ & 2.9619 & 1.7751 & -0.0242 \\
\hline Species & Ulmus alata & 1.00 & 24.00 & $\mathrm{e}^{\left(\mathrm{BO}+\left(\ln (\mathrm{DBH})^{*} \mathrm{~B} 1\right)\right)}$ & 1.5054 & 0.7179 & \\
\hline Species & Ulmus americana & 1.00 & 38.00 & $\mathrm{~B} 0+(\mathrm{DBH} * \mathrm{~B} 1)+\left(\mathrm{DBH}^{2} * \mathrm{~B} 2\right)$ & 5.642 & 2.0847 & -0.0193 \\
\hline Species & Ulmus crassifolia & 1.00 & 17.00 & $\mathrm{~B} 0+(\mathrm{DBH} * \mathrm{~B} 1)+\left(\mathrm{DBH}^{2} * \mathrm{~B} 2\right)$ & 3.8747 & 1.9785 & -0.0015 \\
\hline Species & Ulmus pumila & 1.00 & 36.00 & $\mathrm{~B} 0+(\mathrm{DBH} * \mathrm{~B} 1)$ & 2.7381 & 1.6825 & \\
\hline Species & Ulmus rubra & 1.10 & 37.00 & $\mathrm{e}^{(\mathrm{BO}+(\ln (\mathrm{DBH}) * \mathrm{~B} 1))}$ & 2.1143 & 0.4987 & \\
\hline
\end{tabular}

